\title{
A novel 2-DOF planar translational mechanism composed by scissor-like elements
}

\author{
Yi Yang, Yaping Tian, Yan Peng, and Huayan Pu \\ School of Mechatronic Engineering and Automation, Shanghai University, Shanghai, 200444, China \\ Correspondence to: Yan Peng (pengyan@shu.edu.cn)
}

Received: 20 February 2017 - Accepted: 9 May 2017 - Published: 22 June 2017

\begin{abstract}
The kinematic chain comprised by SLEs (scissor-like elements) has a wide range of motion, which provides a benefit for the mechanism design. A family of SLE-Pa (SLE-parallel) legs which consist of two identical SLE limbs are proposed in this paper. The mobility and kinematics are discussed for three kinds of SLE-Pa legs which are distinguished by the different positions of the middle links in legs. Through assembling these SLE-Pa legs, a novel 2-DOF planar translational mechanism is developed and its work space is studied. For the purpose of adding the recovery function, the elastic elements are installed for this mechanism. The stiffness synthesis of the mechanism is investigated for the various elastic elements and their positions. The approximation of the stiffness coefficient is also derived. Further, this kind of mechanism is applied for the design of the passive docking device. The docking procedure is simulated by Adams, and the prototype of one SLE-Pa leg is presented at the end.
\end{abstract}

\section{Introduction}

The simplest structure of two degrees-of-freedom (DOF) planar translational mechanism is designed by 2-PP (prismaticprismatic) limb (Dong et al., 2004). To reduce the difficulty of manufacturing, enlarge the work space of the end-effector and improve the stiffness of the mechanism, various translational mechanisms by different kinds of kinematic limbs are constructed. Liu and Wang (2003) and Liu et al. (2004) proposed a family of 2-PPa (prismatic-parallelogram) translational mechanisms based on the parallelogram and optimally designed a PRRRP (prismatic-revolute-revolute-revoluteprismatic) 2-DOF parallel mechanism by the utilization of a performance chart (Liu et al., 2006). Wu et al. (2007) also did research on the optimal design of 2-DOF planar parallel mechanism. Kim (2007) and Pham and Kim (2013) developed two types of planar translational parallel manipulators for high-speed positioning applications. Many kinds of 2-DOF planar translational mechanisms are recommended for the design of pick-and-place robots. Huang et al. (2004, 2013) proposed a 2-D version of the delta robot with two sets of parallelograms and studied the optimal design of these 2-DOF translational parallel robots. Generally, most of the above kinematic limbs comprising the 2-DOF planar transla- tional mechanisms were serial kinematic chains or equivalent serial kinematic chains.

The scissor-like element (SLE) is one of the most widely used units in the design of mechanisms, especially for deployable structures. The simplest planar SLE consists of two rigid segments with a revolute joint at their midpoints. The kinematic chain comprised by SLEs has a wide range of motion, which provides a benefit for the mechanism design. In the 1960s, a Spanish architect, E. P. Pienro (Escrig and Valcarcel, 1993; Kaveh and Davaran, 1996), initially employed SLEs to construct the movable theater. After that, SLEs were gradually applied from small-scale structures (Rosenfeld and Logcher, 1988; Escrig et al., 1996) to aerospace structures (Langbecker, 1999). In academic research, You and Pellegrino (1997) presented a general type of two-dimensional foldable structure consisting of different kinds of SLEs. Zhao et al. (2011) constructed foldable stairs with scissor-shape mechanisms. Bai et al. (2013) combined pantograph elements to construct scaling mechanisms for geometric figures. Kaveh et al. (1999) studied the kinematically optimal design of pantograph foldable structures. Dai and Rees (1999), Wei et al. (2010), Ding (2011), and Lu et al. (2017) analyzed the mobility of the foldable structures by screw theory from 


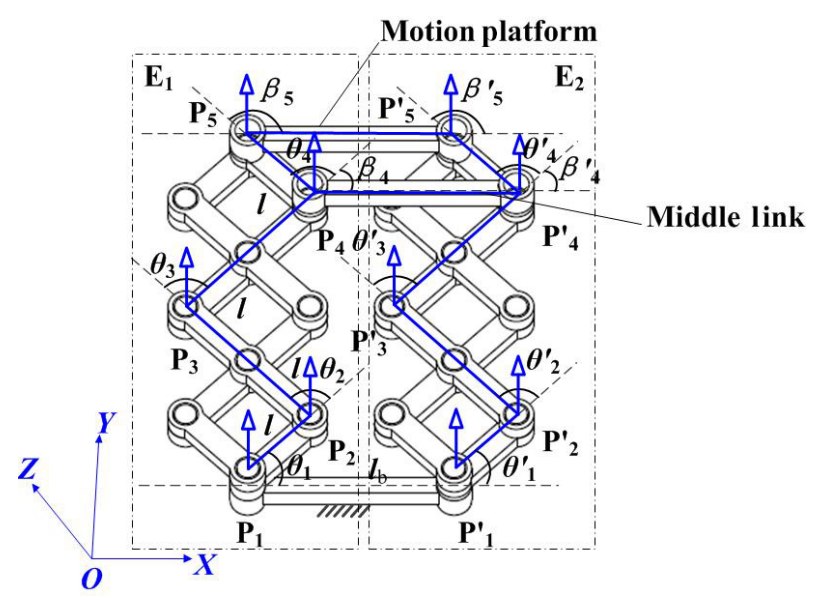

Figure 1. Structure of novel SLE-Pa leg in case 1.

the viewpoint of kinematics. All the achievements with SLEs were mainly focused on deployable mechanisms.

By the utilization of SLEs, we create a novel parallel kinematic chain, which has 2 translational degrees of freedom. Through assembling several of these kinematic chains, a translational parallel mechanism with SLEs is proposed. Based on that, a new passive docking device is invented for the purpose of joining two water pipes aboard a USV (unmanned surface vehicle). With the requirement of the recovery force in the device, the elastic components are set to this translational parallel mechanism. Hence, the stiffness of the platform in the mechanism is investigated under several conditions of the elastic components. Finally, the simulation of the docking procedure is conducted and the prototype of one SLE-Pa leg is presented.

\section{Mobility of novel SLE-Pa leg}

The novel kinematic chain comprises two identical SLE limbs, i.e., limbs $E_{1}$ and $E_{2}$, as shown in Fig. 1. Each SLE limb contains two full SLEs in the middle of the limb and two half-SLEs at both ends of the limb. The length of the link of the full SLE is $2 l$, and one of the links of the halfSLE is $l$. Nodes $P_{1}$ and $P_{5}$ in limb $E_{1}$ are linked by nodes $P_{1}^{\prime}$ and $P_{5}^{\prime}$ in limb $E_{2}$, respectively. The length of $P_{1} P_{1}^{\prime}$ and $P_{5} P_{5}^{\prime}$ is both $l_{b}$. Suppose link $P_{1} P_{1}^{\prime}$ is fixed to the ground and link $P_{5} P_{5}^{\prime}$ is regarded as the motion platform. There is one middle link that connects the two corresponding nodes in the two SLE limbs by hinged joints. This kind of kinematic chain is called SLE-Pa (SLE-parallel) leg. The mobility and kinematics are discussed in the following three cases in relation to the hinged positions of the middle link.

\subsection{Case 1: middle link connecting $P_{4}$ and $P_{4}^{\prime}$}

Screw theory is applied to analyze the mobility of motion platform $P_{5} P_{5}^{\prime}$ as shown in Fig. 1. For SLE limb $E_{1}$, the
Cartesian coordinates of joints $P_{1}, P_{2}, P_{3}, P_{4}$ and $P_{5}$ can be obtained as

$\mathbf{p}_{1}=\left[\begin{array}{c}x_{a} \\ y_{a} \\ 0\end{array}\right] \quad \mathbf{p}_{2}=\left[\begin{array}{c}x_{a}+l_{1} \cos \theta_{1} \\ y_{a}+l_{1} \sin \theta_{1} \\ 0\end{array}\right]$
$\mathbf{p}_{3}=\left[\begin{array}{c}x_{a}+l_{1} \cos \theta_{1}+2 l_{1} \cos \left(\theta_{1}+\theta_{2}\right) \\ y_{a}+l_{1} \sin \theta_{1}+2 l_{1} \sin \left(\theta_{1}+\theta_{2}\right) \\ 0\end{array}\right]$

$\mathbf{p}_{4}=\left[\begin{array}{c}x_{a}+l_{1} \cos \theta_{1}+2 l_{1} \cos \left(\theta_{1}+\theta_{2}\right)+2 l_{1} \cos \left(\theta_{1}+\theta_{2}-\theta_{3}\right) \\ y_{a}+l_{1} \sin \theta_{1}+2 l_{1} \sin \left(\theta_{1}+\theta_{2}\right)+2 l_{1} \sin \left(\theta_{1}+\theta_{2}-\theta_{3}\right) \\ 0\end{array}\right]$

$\mathbf{p}_{5}=\left[\begin{array}{c}x_{a}+l_{1} \cos \theta_{1}+2 l_{1} \cos \left(\theta_{1}+\theta_{2}\right)+2 l_{1} \cos \left(\theta_{1}+\theta_{2}-\theta_{3}\right) \\ +l_{1} \cos \left(\theta_{1}+\theta_{2}-\theta_{3}+\theta_{4}\right) \\ y_{a}+l_{1} \sin \theta_{1}+2 l_{1} \sin \left(\theta_{1}+\theta_{2}\right)+2 l_{1} \sin \left(\theta_{1}+\theta_{2}-\theta_{3}\right) \\ +l_{1} \sin \left(\theta_{1}+\theta_{2}-\theta_{3}+\theta_{4}\right) \\ 0\end{array}\right]$.

Limb $E_{2}$ has the same structure as limb $E_{1}$. The positions of joints in $E_{2}$ appear to be the results of the translation of the joints from $E_{1}$. The Cartesian coordinates of joints can be calculated as

$\mathbf{p}_{i}^{\prime}=\mathbf{p}_{i}+\left[\begin{array}{c}l_{b} \\ 0 \\ 0\end{array}\right] \quad i=1,2, \ldots, 5$.

The axis orientation of each joint is normal to the plane $X O Y$, i.e., $\mathbf{s}_{j}=\left(\begin{array}{lll}0 & 0 & 1\end{array}\right)$. The screws of the above joints are given as

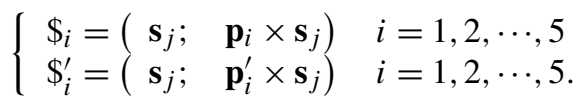

We choose linkages $P_{1} P_{2} P_{3} P_{4} P_{4}^{\prime} P_{3}^{\prime} P_{2}^{\prime} P_{1}^{\prime}$ and $P_{1} P_{2} P_{3} P_{4} P_{5} P_{5}^{\prime} P_{4}^{\prime} P_{3}^{\prime} P_{2}^{\prime} P_{1}^{\prime}$ to establish the screw-loop equations. For the first linkage, the screw-loop equation is

$$
\begin{aligned}
& \$_{1} \dot{\theta}_{1}+\$_{2} \dot{\theta}_{2}-\$_{3} \dot{\theta}_{3}-\$_{4} \dot{\beta}_{4} \\
& =\$_{1}^{\prime} \dot{\theta}_{1}^{\prime}+\$_{2}^{\prime} \dot{\theta}_{2}^{\prime}-\$_{3}^{\prime} \dot{\theta}_{3}^{\prime}-\$_{4}^{\prime} \dot{\beta}_{4}^{\prime} .
\end{aligned}
$$

According to the feature of SLE limbs, the following constraints can be obtained:

$\theta_{2}=\theta_{3}=\theta_{4}, \quad \theta_{2}^{\prime}=\theta_{3}^{\prime}=\theta_{4}^{\prime}, \quad \dot{\theta}_{2}=\dot{\theta}_{3}=\dot{\theta}_{4}$,

$\dot{\theta}_{2}^{\prime}=\dot{\theta}_{3}^{\prime}=\dot{\theta}_{4}^{\prime}$.

Substituting Eq. (4) into Eq. (3), the screw-loop equation can be rewritten as

$$
\begin{aligned}
& \$_{1} \dot{\theta}_{1}+\left(\$_{2}-\$_{3}\right) \dot{\theta}_{2}-\$_{4} \dot{\beta}_{4} \\
& \quad=\$_{1}^{\prime} \dot{\theta}_{1}^{\prime}+\left(\$_{2}^{\prime}-\$_{3}^{\prime}\right) \dot{\theta}_{2}^{\prime}-\$_{4}^{\prime} \dot{\beta}_{4}^{\prime} .
\end{aligned}
$$


In the same way, the screw-loop equation of linkage $P_{1} P_{2} P_{3} P_{4} P_{5} P_{5}^{\prime} P_{4}^{\prime} P_{3}^{\prime} P_{2}^{\prime} P_{1}^{\prime}$ can be derived as

$\$_{1} \dot{\theta}_{1}+\left(\$_{2}-\$_{3}+\$_{4}\right) \dot{\theta}_{2}-\$_{5} \dot{\beta}_{5}$

$$
=\$_{1}^{\prime} \dot{\theta}_{1}^{\prime}+\left(\$_{2}^{\prime}-\$_{3}^{\prime}+\$_{4}^{\prime}\right) \dot{\theta}_{2}^{\prime}-\$_{5}^{\prime} \dot{\beta}_{5}^{\prime} \text {. }
$$

The above Eqs. (5) and (6) can be rearranged in matrix form as

$$
\left[\begin{array}{ccccr}
\$_{1} & \left(\$_{2}-\$_{3}\right) & -\$_{4} & 0 & -\$_{1}^{\prime} \\
& -\left(\$_{2}^{\prime}-\$_{3}^{\prime}\right) & \$_{4}^{\prime} & 0 & - \\
\$_{1} & \left(\$_{2}-\$_{3}+\$_{4}\right) & 0 & -\$_{5} & -\$_{1}^{\prime} \\
& -\left(\$_{2}^{\prime}-\$_{3}^{\prime}+\$_{4}^{\prime}\right) & 0 & \$_{5}^{\prime} &
\end{array}\right]\left[\begin{array}{c}
\dot{\theta}_{1} \\
\dot{\theta}_{2} \\
\dot{\beta}_{4} \\
\dot{\beta}_{5} \\
\dot{\theta}_{1}^{\prime} \\
\dot{\theta}_{2}^{\prime} \\
\dot{\beta}_{4}^{\prime} \\
\dot{\beta}_{5}^{\prime}
\end{array}\right]=0 .
$$

By solving Eq. (7), the velocities of joints are expressed as

$$
\left[\begin{array}{c}
\dot{\theta}_{1} \\
\dot{\theta}_{2} \\
\dot{\beta}_{4} \\
\dot{\beta}_{5} \\
\dot{\theta}_{1}^{\prime} \\
\dot{\theta}_{2}^{\prime} \\
\dot{\beta}_{4}^{\prime} \\
\dot{\beta}_{5}^{\prime}
\end{array}\right]=q_{1}\left[\begin{array}{l}
0 \\
1 \\
0 \\
1 \\
0 \\
1 \\
0 \\
1
\end{array}\right]+q_{2}\left[\begin{array}{c}
1 \\
-1 \\
1 \\
0 \\
1 \\
-1 \\
1 \\
0
\end{array}\right],
$$

where $q_{1}$ and $q_{2}$ are arbitrary real numbers. The result indicates that the mobility of this linkage is 2 and that the velocities of joints are subject to the following relationship:

$\dot{\theta}_{1}=\dot{\theta}_{1}^{\prime}=\dot{\beta}_{4}=\dot{\beta}_{4}^{\prime}, \quad \dot{\theta}_{2}=\dot{\theta}_{2}^{\prime}, \quad \dot{\beta}_{5}=\dot{\beta}_{5}^{\prime}=\dot{\theta}_{1}+\dot{\theta}_{2}$.

Equation (9) shows that the two SLE limbs are always parallel. Further, the kinematic screw of the platform $P_{5} P_{5}^{\prime}$ can be written as

$\$_{p}=\$_{1} \dot{\theta}_{1}+\left(\$_{2}-\$_{3}+\$_{4}\right) \dot{\theta}_{2}-\$_{5} \dot{\beta}_{5}$.

Substituting Eq. (8) into Eq. (10), the kinematic screw of the platform $P_{5} P_{5}^{\prime}$ can be derived as

$\$_{p}=q_{1}\left[\begin{array}{c}0 \\ 0 \\ 0 \\ -\sin \left(\theta_{1}+\theta_{2}\right) \\ \cos \left(\theta_{1}+\theta_{2}\right) \\ 0\end{array}\right]+q_{2}\left[\begin{array}{c}0 \\ 0 \\ 0 \\ -\sin \theta_{1} \\ \cos \theta_{1} \\ 0\end{array}\right]$.

The corresponding reciprocal screw of the platform can be derived as

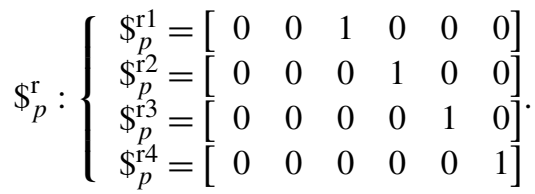

Equations (11) and (12) indicates that the motion platform of the SLE-Pa leg has 2 pure translational degrees of freedom.

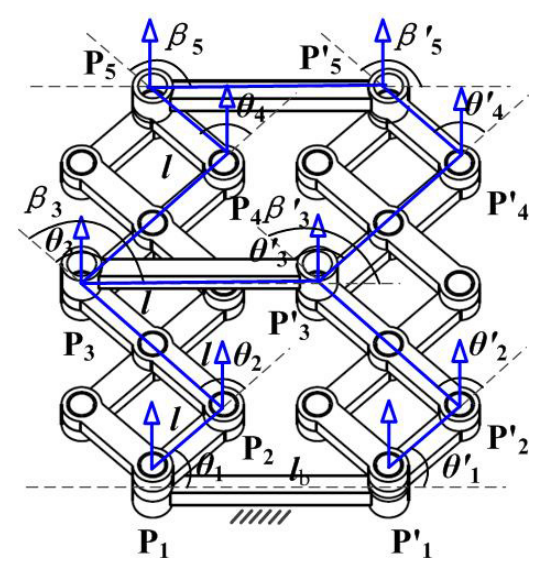

Figure 2. Structure of novel SLE-Pa leg in case 2.

\subsection{Case 2: middle link connecting $P_{3}$ and $P_{3}^{\prime}$}

In this case, the middle link connects $P_{3}, P_{3}^{\prime}$ between two SLE limbs, as shown in Fig. 2. The screw-loop equations are established for linkages $P_{1} P_{2} P_{3} P_{3}^{\prime} P_{2}^{\prime} P_{1}^{\prime}$ and $P_{1} P_{2} P_{3} P_{4} P_{5} P_{5}^{\prime} P_{4}^{\prime} P_{3}^{\prime} P_{2}^{\prime} P_{1}^{\prime}$ with a consideration of the features of SLE limb, as follows:

$$
\left\{\begin{array}{c}
\$_{1} \dot{\theta}_{1}+\$_{2} \dot{\theta}_{2}-\$_{3} \dot{\beta}_{3}=\$_{1}^{\prime} \dot{\theta}_{1}^{\prime}+\$_{2}^{\prime} \dot{\theta}_{2}^{\prime}-\$_{3}^{\prime} \dot{\beta}_{3}^{\prime} \\
\$_{1} \dot{\theta}_{1}+\left(\$_{2}-\$_{3}+\$_{4}\right) \dot{\theta}_{2}-\$_{5} \dot{\beta}_{5} \\
=\$_{1}^{\prime} \dot{\theta}_{1}^{\prime}+\left(\$_{2}^{\prime}-\$_{3}^{\prime}+\$_{4}^{\prime} \dot{\theta}_{2}^{\prime}-\$_{5}^{\prime} \dot{\beta}_{5}^{\prime}\right.
\end{array}\right.
$$

The above Eq. (13) can be rewritten in a matrix form as

$$
\left[\begin{array}{ccccc}
\$_{1} & \$_{2} & -\$_{3} & 0 & -\$_{1}^{\prime} \\
& -\$_{2}^{\prime} & \$_{3}^{\prime} & 0 & \\
\$_{1} & \left(\$_{2}-\$_{3}+\$_{4}\right) & 0 & -\$_{5} & -\$_{1}^{\prime} \\
& -\left(\$_{2}^{\prime}-\$_{3}^{\prime}+\$_{4}^{\prime}\right) & 0 & \$_{5}^{\prime} &
\end{array}\right]\left[\begin{array}{c}
\dot{\theta}_{1} \\
\dot{\theta}_{2} \\
\dot{\beta}_{3} \\
\dot{\beta}_{5} \\
\dot{\theta}_{1}^{\prime} \\
\dot{\theta}_{2}^{\prime} \\
\dot{\beta}_{3}^{\prime} \\
\dot{\beta}_{5}^{\prime}
\end{array}\right]=0
$$

According to Eq. (14), the velocities of joints are obtained as

$$
\left[\begin{array}{c}
\dot{\theta}_{1} \\
\dot{\theta}_{2} \\
\dot{\beta}_{3} \\
\dot{\beta}_{5} \\
\dot{\theta}_{1}^{\prime} \\
\dot{\theta}_{2}^{\prime} \\
\dot{\beta}_{3}^{\prime} \\
\dot{\beta}_{5}^{\prime}
\end{array}\right]=q_{1}\left[\begin{array}{c}
-1 \\
1 \\
0 \\
0 \\
-1 \\
1 \\
0 \\
0
\end{array}\right]+q_{2}\left[\begin{array}{l}
1 \\
0 \\
1 \\
1 \\
1 \\
0 \\
1 \\
1
\end{array}\right] \text {. }
$$

Equation (15) shows that the mobility of this linkage is 2, and the velocities of joints are also subject to the following relationship:

$\dot{\theta}_{1}=\dot{\theta}_{1}^{\prime}, \quad \dot{\theta}_{2}=\dot{\theta}_{2}^{\prime}, \quad \dot{\beta}_{3}=\dot{\beta}_{3}^{\prime}=\dot{\beta}_{5}=\dot{\beta}_{5}^{\prime}=\dot{\theta}_{1}+\dot{\theta}_{2}$. 


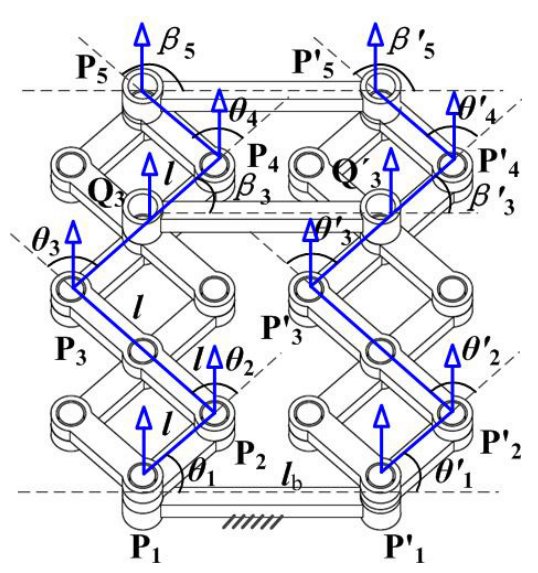

Figure 3. Structure of novel SLE-Pa leg in case 3.

The two SLEs are always parallel as well. The kinematic screw of the platform can be calculated as

$\$_{p}=q_{1}\left[\begin{array}{c}0 \\ 0 \\ 0 \\ -\sin \left(\theta_{1}+\theta_{2}\right) \\ \cos \left(\theta_{1}+\theta_{2}\right) \\ 0\end{array}\right]+q_{2}\left[\begin{array}{c}0 \\ 0 \\ 0 \\ -\sin \theta_{1} \\ \cos \theta_{1} \\ 0\end{array}\right]$.

The corresponding reciprocal screw of the platform can be calculated as

$$
\$_{p}^{\mathrm{r}}:\left\{\begin{array}{l}
\$_{p}^{\mathrm{r} 1}=\left[\begin{array}{llllll}
0 & 0 & 1 & 0 & 0 & 0
\end{array}\right] \\
\$_{p}^{\mathrm{r} 2}=\left[\begin{array}{llllll}
0 & 0 & 0 & 1 & 0 & 0
\end{array}\right] \\
\$_{p}^{\mathrm{r} 3}=\left[\begin{array}{llllll}
0 & 0 & 0 & 0 & 1 & 0
\end{array}\right] \\
\$_{p}^{\mathrm{r} 4}=\left[\begin{array}{llllll}
0 & 0 & 0 & 0 & 0 & 1
\end{array}\right]
\end{array}\right.
$$

It indicates that the motion platform has 2 pure translational degrees of freedom, which is the same with the results of case 1 .

\subsection{Case 3: middle link connecting $Q_{3}$ and $Q_{3}^{\prime}$}

As shown in Fig. 3, the screw-loop equations are established for linkages $P_{1} P_{2} P_{3} Q_{3} Q_{3}^{\prime} P_{3}^{\prime} P_{2}^{\prime} P_{1}^{\prime}$ and $P_{1} P_{2} P_{3} P_{4} P_{5} P_{5}^{\prime} P_{4}^{\prime} P_{3}^{\prime} P_{2}^{\prime} P_{1}^{\prime}$. By the solution of these screw-loop equations, the velocities of joints can be obtained as

$$
\left[\begin{array}{c}
\dot{\theta}_{1} \\
\dot{\theta}_{2} \\
\dot{\beta}_{3} \\
\dot{\beta}_{5} \\
\dot{\theta}_{1}^{\prime} \\
\dot{\theta}_{2}^{\prime} \\
\dot{\beta}_{3}^{\prime} \\
\dot{\beta}_{5}^{\prime}
\end{array}\right]=q_{1}\left[\begin{array}{l}
0 \\
1 \\
0 \\
1 \\
0 \\
1 \\
0 \\
1
\end{array}\right]+q_{2}\left[\begin{array}{c}
1 \\
-1 \\
1 \\
0 \\
1 \\
-1 \\
1 \\
0
\end{array}\right]
$$

$$
+q_{3}\left[\begin{array}{c}
\left(2 l_{1} \sin \theta_{2}-l_{b} \sin \left(\theta_{1}+\theta_{2}\right)\right) /\left(-l_{b} \sin \theta_{1}\right) \\
\left(l_{1} \sin \theta_{2}+l_{b} \sin \left(\theta_{1}+\theta_{2}\right)\right) /\left(-l_{b} \sin \theta_{1}\right) \\
\sin \left(\theta_{1}+\theta_{2}\right) / \sin \theta_{1} \\
0 \\
2 l_{1} \sin \theta_{2} /\left(-l_{b} \sin \theta_{1}\right) \\
0 \\
\left(l_{1} \sin \theta_{2}-l_{b} \sin \theta_{1}\right) /\left(-l_{b} \sin \theta_{1}\right) \\
1
\end{array}\right] .
$$

Equation (19) shows that this SLE-Pa leg has three mobilities. Furthermore, the corresponding reciprocal screw of the platform can be calculated as

$$
\$_{p}^{\mathbf{r}}:\left\{\begin{array}{l}
\$_{p}^{r 1}=\left[\begin{array}{llllll}
0 & 0 & 1 & 0 & 0 & 0
\end{array}\right] \\
\$_{p}^{r 2}=\left[\begin{array}{llllll}
0 & 0 & 0 & 1 & 0 & 0
\end{array}\right] \\
\$_{p}^{r 3}=\left[\begin{array}{llllll}
0 & 0 & 0 & 0 & 1 & 0
\end{array}\right]
\end{array}\right.
$$

Equation (20) indicates that the motion platform has an extra rotational degree of freedom along the $z$ axis, in addition to the 2 translational degrees of freedom.

So far, the mobilities of these kinds of SLE-Pa legs in three cases have been investigated based on screw theory. The results of the other SLE-Pa legs can be deduces from the above three cases. The results are listed in Table 1.

By observing Table 1, we can conclude that if the middle link connects two corresponding corner joints of the SLE limbs, the two SLE limbs are always parallel and the motion platform has 2 pure translational degrees of freedom. If the middle link connects two corresponding middle joints of the SLE limbs, the motion platform has 3 DOFs, which includes 2 translational degrees of freedom and 1 rotational degree of freedom along the $z$ axis.

In cases 1 and 2, suppose

$$
\frac{\sin \theta_{1}}{\sin \left(\theta_{1}+\theta_{2}\right)}=\frac{\cos \theta_{1}}{\cos \left(\theta_{1}+\theta_{2}\right)}
$$

and substitute it into Eqs. (11) or (17). It is found that the mobility of the SLE-Pa leg reduces to 1 . At this moment, the SLE-Pa leg is at the singularity. Solving Eq. (21), the singular angle is

$\theta_{2}=0$ or $\pi$,

which corresponds to the maximum or minimum length configuration of the SLE limb.

\section{Kinematic analysis of novel 2-DOF translational mechanism}

Through assembling the above SLE-Pa legs, a novel 2-DOF translational mechanism is invented. The 2-DOF translational mechanism is composed of three SLE-Pa legs, which are distributed around the center point by $120^{\circ}$, as shown in Fig. 4. As mentioned above, it is easily established that the mobility of this parallel mechanism is 2 . 
Table 1. Mobility of SLE-Pa legs.

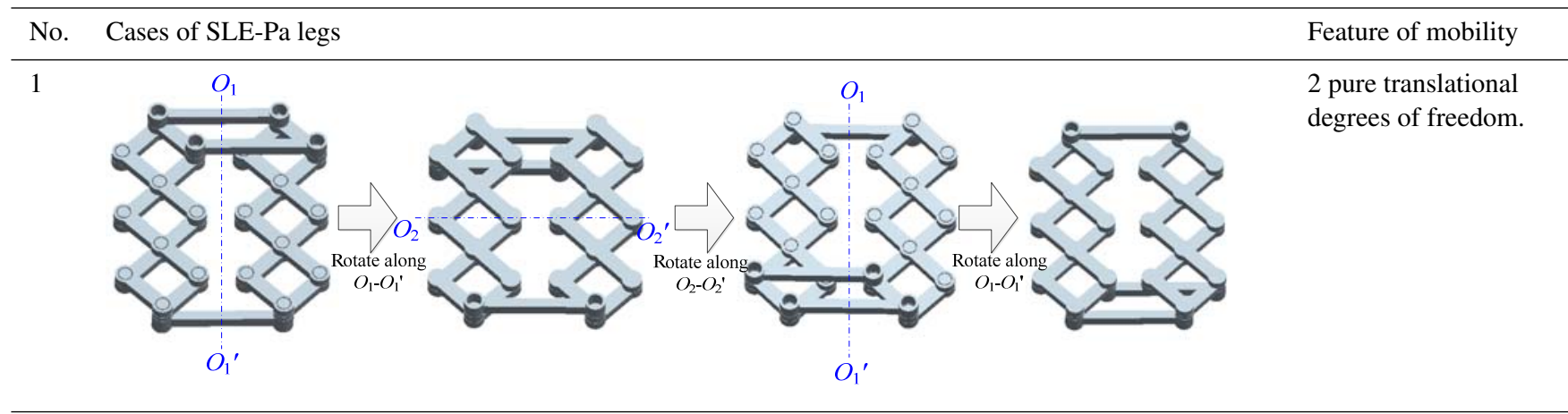

2

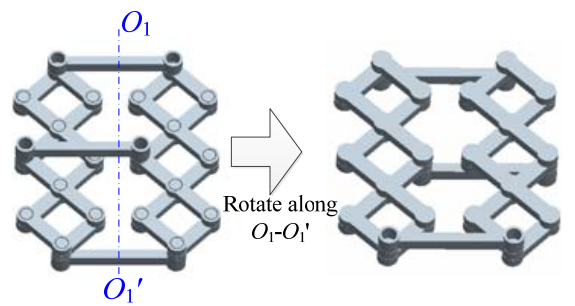

2 pure translational degrees of freedom.

3

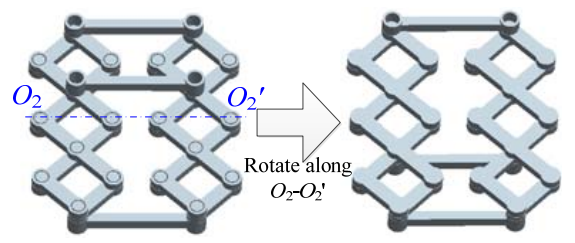

2 translational degrees of freedom +1 rotational degree of freedom along $z$ axis

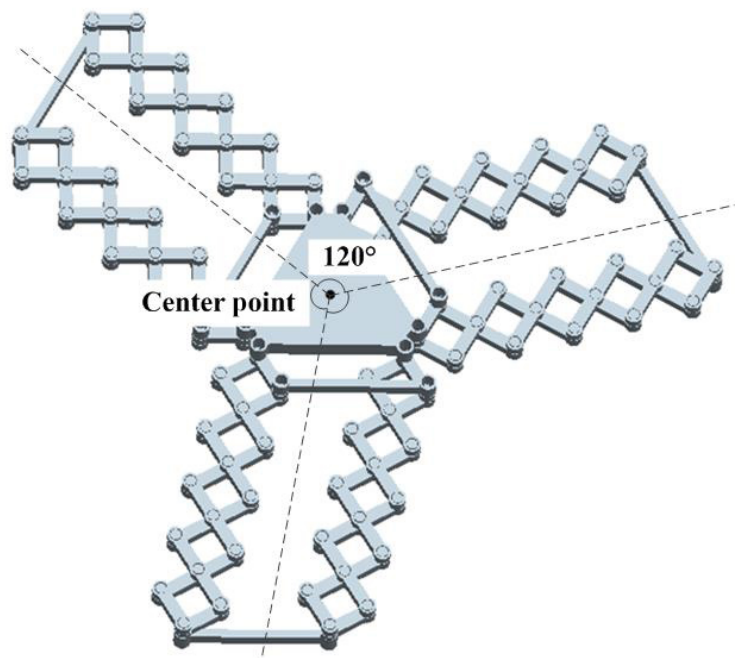

Figure 4. Two-DOF translational parallel mechanism.
In order to simplify the analysis of the kinematics, we substitute an RPR (revolute-prismatic-revolute) limb into the SLE-Pa leg and restrict the $z$-axis rotational degree of freedom of the motion platform. This results in the motion platform only having 2 translational degrees of freedom, which agrees with the feature of the original mechanism. The schematic diagram is found in Fig. 5.

For the kinematic chain $B_{1 \mathrm{E}} A_{1 \mathrm{E}},\left(x_{B_{1 \mathrm{E}}}, y_{B_{1 \mathrm{E}}}\right),\left(x_{A_{1 \mathrm{E}}}, y_{A_{1 \mathrm{E}}}\right)$ is its initial position. When this mechanism moves, the new coordinates of the platform are $\left(x_{B_{1 \mathrm{E}}}+p_{x}, y_{B_{1 \mathrm{E}}}+p_{y}\right)$. The displacement equations of one RPR kinematic chain can be obtained as

$$
\left\{\begin{array}{l}
\left(x_{B_{1 \mathrm{E}}}+p_{x}\right)-{ }^{1} H_{1} \cos ^{1} \theta_{1}=x_{A_{1 \mathrm{E}}} \\
\left(y_{B_{1 \mathrm{E}}}+p_{y}\right)-{ }^{1} H_{1} \sin ^{1} \theta_{1}=y_{A_{1 \mathrm{E}}}
\end{array} .\right.
$$

The displacement ${ }^{1} H_{1}$ and angle ${ }^{1} \theta_{1}$ can be derived as

$\left\{\begin{array}{l}{ }^{1} H_{1}=\sqrt{\left(x_{B_{1 \mathrm{E}}}+p_{x}-x_{A_{1 \mathrm{E}}}\right)^{2}+\left(y_{B_{1 \mathrm{E}}}+p_{y}-y_{A_{1 \mathrm{E}}}\right)^{2}} \\ { }^{1} \theta_{1}=\arccos \left(\left(x_{B_{1 \mathrm{E}}}+p_{x}-x_{A_{1 \mathrm{E}}}\right) /{ }^{1} H_{1}\right)\end{array}\right.$.

Given that the length of each link of the full SLE is $2 l$, the length of the link of half the SLE is $l$. The angle between two 


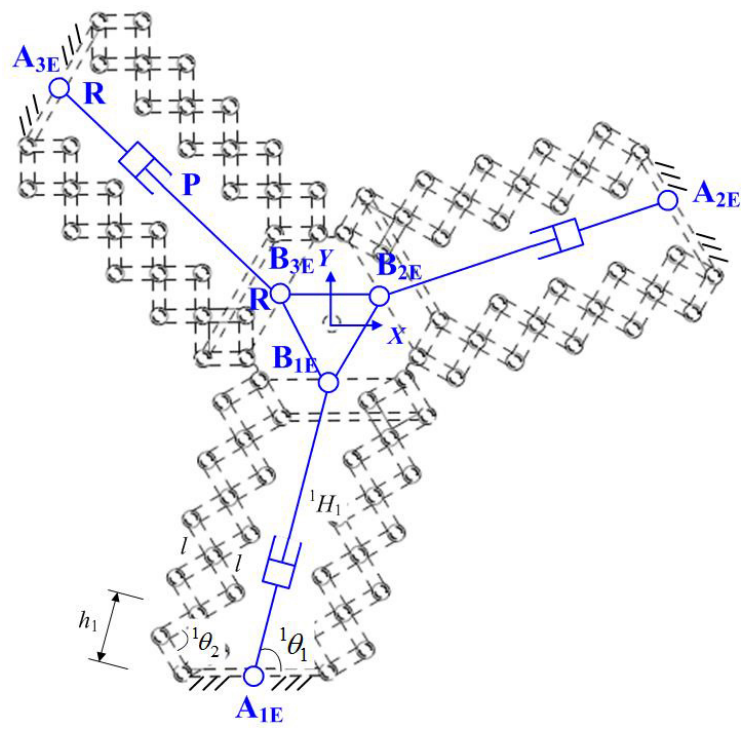

Figure 5. Simplified translational parallel mechanism.

links in $B_{1 \mathrm{E}} A_{1 \mathrm{E}}$ is ${ }^{1} \theta_{2} . s$ is the number of the parallelogram in a single SLE limb. $h_{1}$ is the length of the single parallelogram. Thus, the length of $B_{1 \mathrm{E}} A_{1 \mathrm{E}}$ is

${ }^{1} H_{1}=s h_{1}=2 s l \sin \frac{{ }^{1} \theta_{2}}{2}$.

Substitute the above equation into Eq. (24) gives

$$
\left\{\begin{array}{c}
{ }^{1} \theta_{2}=2 \arcsin \\
\left(\sqrt{\left(x_{B_{1 \mathrm{E}}}+p_{x}-x_{A_{\mathrm{IE}}}\right)^{2}+\left(y_{B_{\mathrm{IE}}}+p_{y}-y_{A_{\mathrm{IE}}}\right)^{2}} /(2 s l)\right) \\
{ }^{1} \theta_{1}=\arccos \left(\left(x_{B_{1 \mathrm{IE}}}+p_{x}-x_{A_{\mathrm{IE}}}\right) /\right. \\
\left.\sqrt{\left(x_{B_{1 \mathrm{E}}}+p_{x}-x_{A_{\mathrm{IE}}}\right)^{2}+\left(y_{B_{\mathrm{IE}}}+p_{y}-y_{A_{\mathrm{IE}}}\right)^{2}}\right)
\end{array} .\right.
$$

Through differentiating the above equations, the velocity equations can be obtained:

$$
\left\{\begin{array}{l}
{ }^{1} \dot{\theta}_{2}=\left(\dot{p}_{x} \cos ^{1} \theta_{1}+\dot{p}_{y} \sin ^{1} \theta_{1}\right) /\left(2 \operatorname{sicos} \frac{{ }^{1} \theta_{2}}{2}\right) \\
{ }^{1} \dot{\theta}_{1}=-\left(\dot{p}_{x} \sin ^{1} \theta_{1}-\dot{p}_{y} \cos ^{1} \theta_{1}\right) /\left(2 \sin \sin \frac{{ }^{1} \theta_{2}}{2}\right)
\end{array} .\right.
$$

They can be expressed in matrix form:

$\mathbf{J}\left[\begin{array}{c}{ }^{1} \dot{\theta}_{1} \\ { }^{1} \dot{\theta}_{2}\end{array}\right]=\left[\begin{array}{c}\dot{P}_{x} \\ \dot{P}_{y}\end{array}\right]$,

where the Jacobian matrix is

$\mathbf{J}=\left[\begin{array}{cc}-2 s l \sin \frac{{ }^{1} \theta_{2}}{2} \sin ^{1} \theta_{1} & 2 s l \cos \frac{{ }^{1} \theta_{2}}{2} \cos ^{1} \theta_{1} \\ 2 s l \sin \frac{{ }^{1} \theta_{2}}{2} \cos ^{1} \theta_{1} & 2 s l \cos \frac{{ }^{1} \theta_{2}}{2} \sin ^{1} \theta_{1}\end{array}\right]$.

The determinant of the matrix is $|J|=-2 s^{2} l^{2} \sin ^{1} \theta_{2}$. Let $|J|=0$; then, the singular configuration can be obtained, i.e., ${ }^{1} \theta_{2}=0$ and $\pi$. This result agrees with Eq. (22).
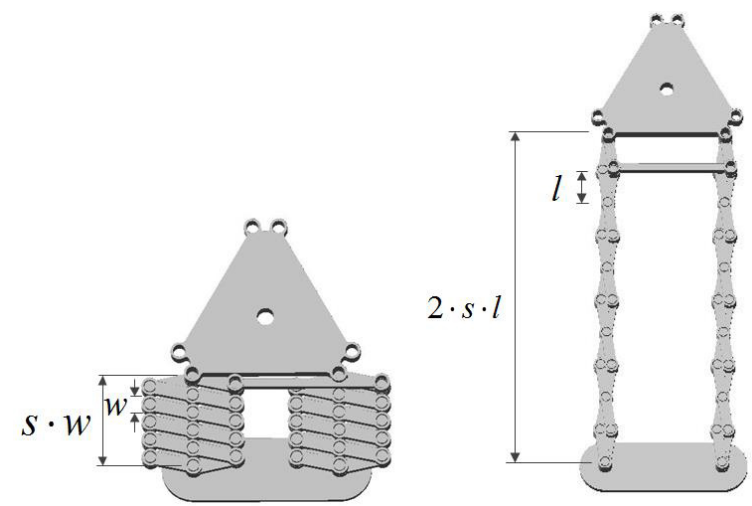

Figure 6. Minimum and maximum length of ${ }^{i} H_{1}$.

For the other kinematic chains, we can calculate the angles by the transformation of coordinates. Given $\alpha_{i}$ is the rotation angle from $B_{1 \mathrm{E}} A_{1 \mathrm{E}}$ to $B_{i \mathrm{E}} A_{i \mathrm{E}}$, the new displacement ${ }^{i} H_{1}$ and angle ${ }^{i} \theta_{1}$ are

$$
\left\{\begin{array}{l}
{ }^{i} H_{1}=\sqrt{\left({ }^{i} C_{x}+p_{x}\right)^{2}+\left({ }^{i} C_{y}+p_{y}\right)^{2}} \\
{ }^{i} \theta_{1}=\arccos \left(\left({ }^{i} C_{x}+p_{x}\right) /{ }^{i} H_{1}\right) \\
{ }^{i} C_{x}\left(x_{B_{1 \mathrm{E}}}-x_{\left.A_{1 \mathrm{E}}\right) \cos \alpha_{i}-\left(\gamma_{B_{1 \mathrm{E}}}-\gamma_{A_{1 \mathrm{E}}}\right) \sin \alpha_{i}}\right. \\
{ }^{i} C_{y}=\left(x_{B_{1 \mathrm{E}}}-x_{A_{1 \mathrm{E}}}\right) \sin \alpha_{i}+\left(\gamma_{B_{1 \mathrm{E}}}-\gamma_{A_{1 \mathrm{E}}}\right) \cos \alpha_{i}
\end{array} .\right.
$$

Given all kinematic chains are identical, the range of each linear motion element is

$H_{\mathrm{lb}} \leq{ }^{i} H_{1} \leq H_{\mathrm{ub}} \quad i=1,2, \ldots, n$,

where $H_{\mathrm{lb}}, H_{\mathrm{ub}}$ are the lower and upper bounds. As shown in Fig. 6, the minimum and maximum length of ${ }^{i} H_{1}$ can be estimated:

$H_{\mathrm{lb}} \approx s \cdot w$
$H_{\mathrm{ub}} \approx 2 \cdot s \cdot l$,

where $w$ is the width of each link.

The work space of the platform is the intersection of the work spaces of each kinematic chain. According to Eq. (30), the work space of each kinematic chain is a torus, whose inner and outer radius are $H_{\mathrm{lb}}$ and $H_{\mathrm{ub}}$, respectively. The center of the torus is $\left(-\left(x_{B_{1 \mathrm{E}}}-x_{A_{1 \mathrm{E}}}\right) \cos \alpha_{i}+\left(y_{B_{1 \mathrm{E}}}-\right.\right.$ $\left.\left.y_{A_{1 \mathrm{E}}}\right) \sin \alpha_{i},-\left(x_{B_{1 \mathrm{E}}}-x_{A_{1 \mathrm{E}}}\right) \sin \alpha_{i}-\left(y_{B_{1 \mathrm{E}}}-y_{A_{1 \mathrm{E}}}\right) \cos \alpha_{i}\right)$, denoted by $D_{i \mathrm{E}}$. The 2-DOF planar mechanism is composed of three identical chains. According to the ratio of $H_{\mathrm{ub}}$ to $H_{\mathrm{lb}}$, there are two types of work spaces.

I. Type I: $H_{\mathrm{ub}} / H_{\mathrm{lb}}>\sqrt{3}$

Given $\left|D_{1 \mathrm{E}} D_{2 \mathrm{E}}\right|=\left|D_{2 \mathrm{E}} D_{3 \mathrm{E}}\right|=\left|D_{3 \mathrm{E}} D_{1 \mathrm{E}}\right|=D D$, the work space of the platform in this type can be classified as consisting of three categories. The shapes of each category can be seen in Fig. 7. The different ranges of the above three categories are displayed by the plot of $D D / H_{\mathrm{lb}}$ vs. $H_{\mathrm{ub}} / H_{\mathrm{lb}}$ in Fig. 8. 


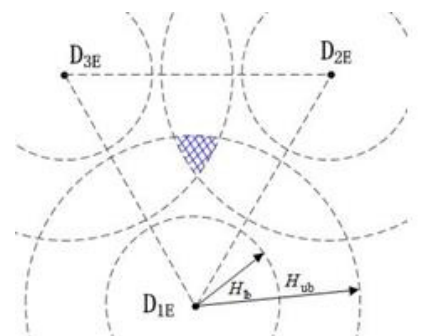

(a)

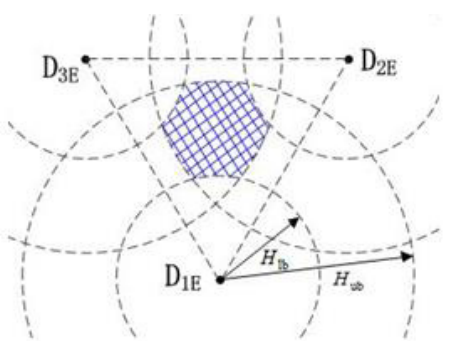

(b)

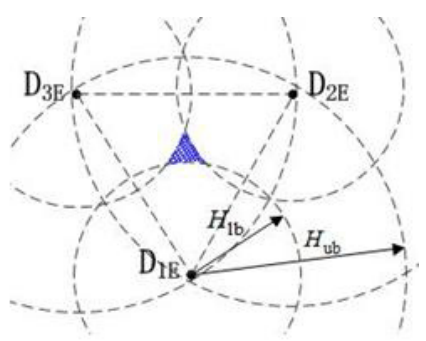

(c)

(a) $\sqrt{3} H_{\mathrm{tb}}>D D \geq\left(\sqrt{3} H_{\mathrm{tb}}+\sqrt{3 H_{\mathrm{tb}}^{2}+4\left(H_{\mathrm{tb}}{ }^{2}-H_{\mathrm{bb}}{ }^{2}\right)}\right) / 2$

(b) $\left(\sqrt{3} H_{1 \mathrm{~b}}+\sqrt{3 H_{\mathrm{b}}^{2}+4\left(H_{\mathrm{w}}{ }^{2}-H_{\mathrm{b}}{ }^{2}\right)}\right) / 2>D D>2 H_{\mathrm{b}}$

(c) $\quad 2 H_{\mathrm{tb}} \geq D D>\sqrt{3} H_{\mathrm{tb}}$

Figure 7. Three categories of type I work space.

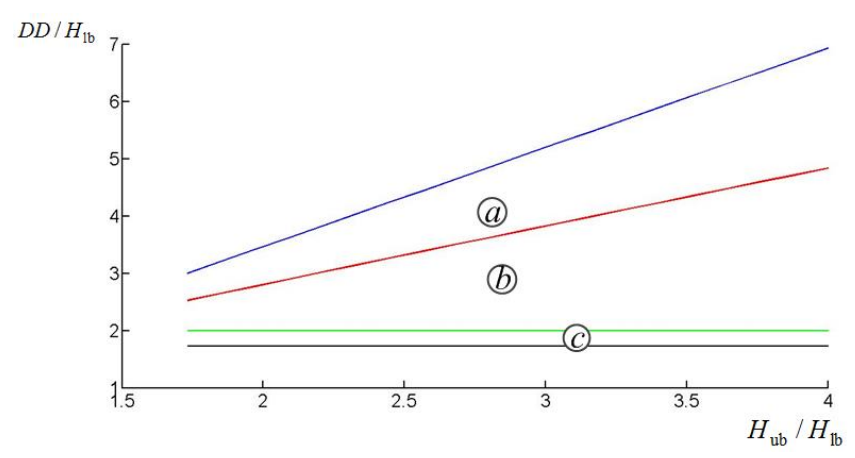

Figure 8. Ranges of three categories of type I.

II. Type II: $1 \leq H_{\mathrm{ub}} / H_{\mathrm{lb}} \leq \sqrt{3}$

In this type, the work space of the platform can also be classified as consisting of three categories, whose shapes are as shown in Fig. 9. The ranges of the above three categories are plotted in Fig. 10.

The numeric value of $\sqrt{3}$ is 1.73 . By using the above results, the work spaces of these kinds of parallel mechanisms can be estimated. For the SLE-Pa leg, the ratio of $H_{\mathrm{ub}}$ to $H_{\mathrm{lb}}$ equals $2 l / w$. In practice, the length-to-width ratio $l / w$ of most links is generally larger than 3.0 , and the ratio $2 l / w$ is definitely more than 1.73. In our models for example, the length $l$ is $27 \mathrm{~mm}$, the width $w$ is $8 \mathrm{~mm}$ and the ratio $2 l / w$ equals 6.75 . Thus, the shapes of most work spaces of these 2-DOF planar translational mechanisms belong to type I. The exact shapes depend on the specific parameters of $H_{\mathrm{lb}}, H_{\mathrm{ub}}$ and the positions of SLE-Pa legs.

\section{Stiffness synthesis}

For the purpose of adding the recovery function, the elastic elements are installed for this mechanism. The various elastic elements and positions result in the different stiffnesses of the motion platform. In this section, we choose a torsional spring and an ordinary cylinder spring as elastic elements to discuss the variation in stiffness.

\subsection{Case 1: torsional springs installed in the corner joints of SLE-Pa legs}

As shown in Fig. 11, the torsional springs are all installed in the corner joints of SLE-Pa legs. Given that stiffness coefficient of each torsional spring is $k_{\theta}$, the total elastic energy of the mechanism is

$V=\sum_{i=1}^{n} \frac{1}{2} k_{\theta}^{i} \theta_{2}^{2}$.

Differentiating Eq. (32) to the displacements $p_{x}$ and $p_{y}$, the corresponding generalized force $Q_{x}$ and $Q_{y}$ of the platform can be calculated by the following equation:

$$
\left\{\begin{array}{l}
Q_{x}=-\frac{\partial V}{\partial p_{x}}=-\sum_{i=1}^{n} k_{\theta} \Delta^{i} \theta_{2} \frac{\partial^{i} \theta_{2}}{\partial p_{x}} \\
Q_{y}=-\frac{\partial V}{\partial p_{y}}=-\sum_{i=1}^{n} k_{\theta} \Delta^{i} \theta_{2} \frac{\partial^{i} \theta_{2}}{\partial p_{y}}
\end{array}\right.
$$

Utilizing Eq. (26), the derivative of ${ }^{i} \theta_{2}$ versus $p_{x}$ and $p_{y}$ can be obtained as

$\frac{\partial^{i} \theta_{2}}{\partial p_{x}}=\frac{\cos ^{i} \theta_{1}}{\cos \frac{i_{2}}{2}} \frac{1}{s l} \quad \frac{\partial^{i} \theta_{2}}{\partial p_{y}}=\frac{\sin ^{i} \theta_{1}}{\cos \frac{i_{2}}{2}} \frac{1}{s l}$.

Continuing to differentiate Eq. (33) to $p_{x}, p_{y}$ and ignoring the second-class derivative of ${ }^{i} \theta_{2}$, we can calculate the stiffness matrix of the platform.

$\mathbf{K}_{\theta}=k_{\theta}\left[\begin{array}{cc}\sum_{i=1}^{n}\left(\frac{\partial^{i} \theta_{2}}{\partial p_{x}}\right)^{2} & \sum_{i=1}^{n}\left(\frac{\partial^{i} \theta_{2}}{\partial p_{x}} \frac{\partial^{i} \theta_{2}}{\partial p_{y}}\right) \\ \sum_{i=1}^{n}\left(\frac{\partial^{i} \theta_{2}}{\partial p_{x}} \frac{\partial^{i} \theta_{2}}{\partial p_{y}}\right) & \sum_{i=1}^{n}\left(\frac{\partial^{i} \theta_{2}}{\partial p_{y}}\right)^{2}\end{array}\right]$ 


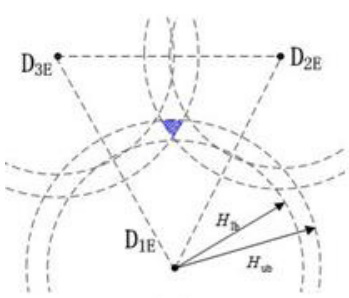

(a)

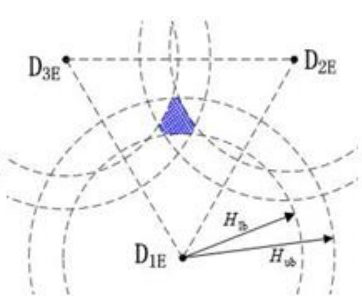

(b)

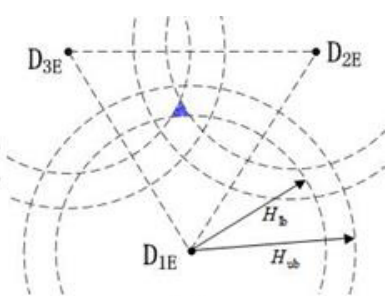

(c)

(a) $\sqrt{3} H_{\mathrm{wb}}>D D \geq\left(\sqrt{3} H_{\mathrm{bb}}+\sqrt{3 H_{\mathrm{bb}}{ }^{2}+4\left(H_{\mathrm{wb}}{ }^{2}-H_{\mathrm{tb}}{ }^{2}\right)}\right) / 2$

(b) $\left(\sqrt{3} H_{\mathrm{tb}}+\sqrt{3 H_{\mathrm{b}}{ }^{2}+4\left(H_{\mathrm{wb}}{ }^{2}-H_{\mathrm{b}}{ }^{2}\right)}\right) / 2>D D>\left(\sqrt{3} H_{\mathrm{wb}}+\sqrt{3 H_{\mathrm{wb}}{ }^{2}-4\left(H_{\mathrm{wb}}{ }^{2}-H_{\mathrm{tb}}{ }^{2}\right)}\right) / 2$

(c) $\left(\sqrt{3} H_{\mathrm{wb}}+\sqrt{3 H_{\mathrm{wb}}^{2}-4\left(H_{\mathrm{wb}}^{2}-H_{\mathrm{tb}}^{2}\right)}\right) / 2 \geq D D>\sqrt{3} H_{\mathrm{tb}}$

Figure 9. Three categories of type II work space.

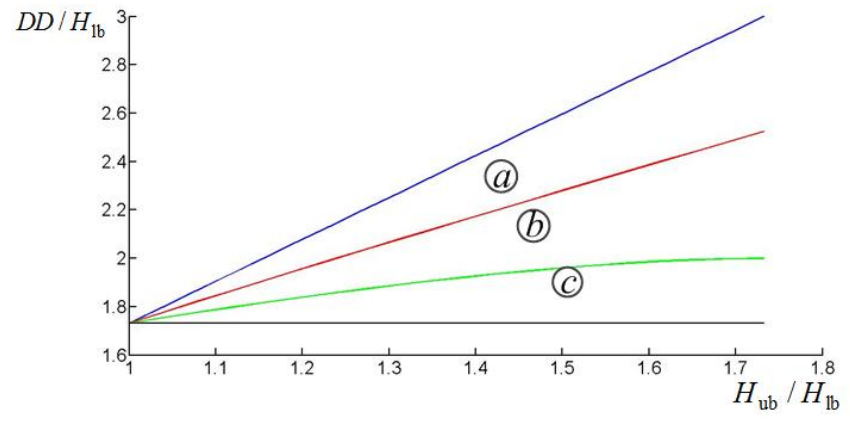

Figure 10. Ranges of three categories of type II.

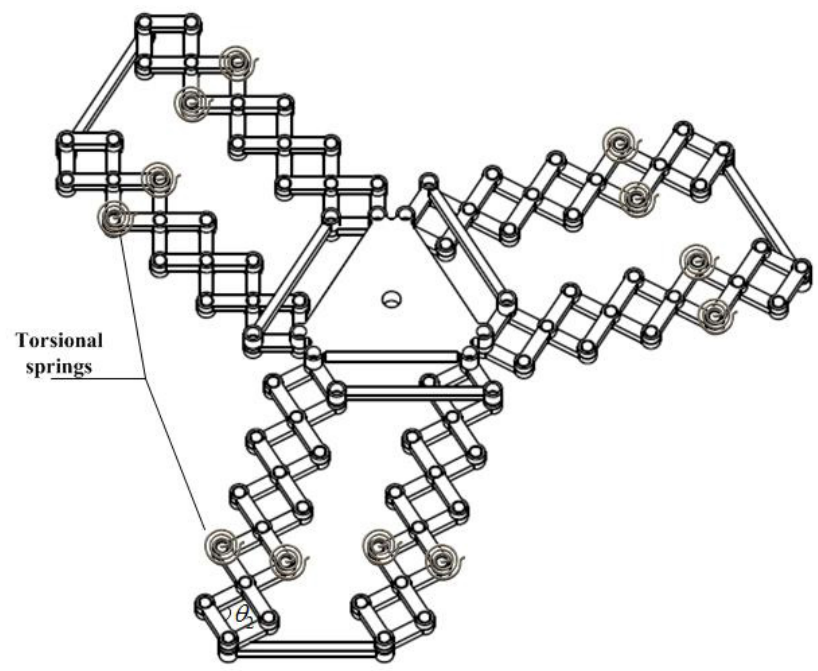

Figure 11. Torsional springs installed in the corner joints.

$$
=\frac{k_{\theta}}{(s l)^{2}}\left[\begin{array}{cc}
\sum_{i=1}^{n} \frac{\cos ^{2}{ }^{2} \theta_{1}}{\cos ^{2} \frac{\theta_{2}}{2}} & \sum_{i=1}^{n} \frac{\cos ^{i} \theta_{1} \sin ^{i} \theta_{1}}{\cos ^{2} \frac{{ }^{i} \theta_{2}}{2}} \\
\sum_{i=1}^{n} \frac{\cos ^{i} \theta_{1} \sin ^{i} \theta_{1}}{\cos ^{2} \frac{{ }^{i}}{2}} & \sum_{i=1}^{n} \frac{\sin ^{2} \theta_{1}}{\cos ^{2} \frac{{ }^{i} \theta_{2}}{2}}
\end{array}\right]
$$

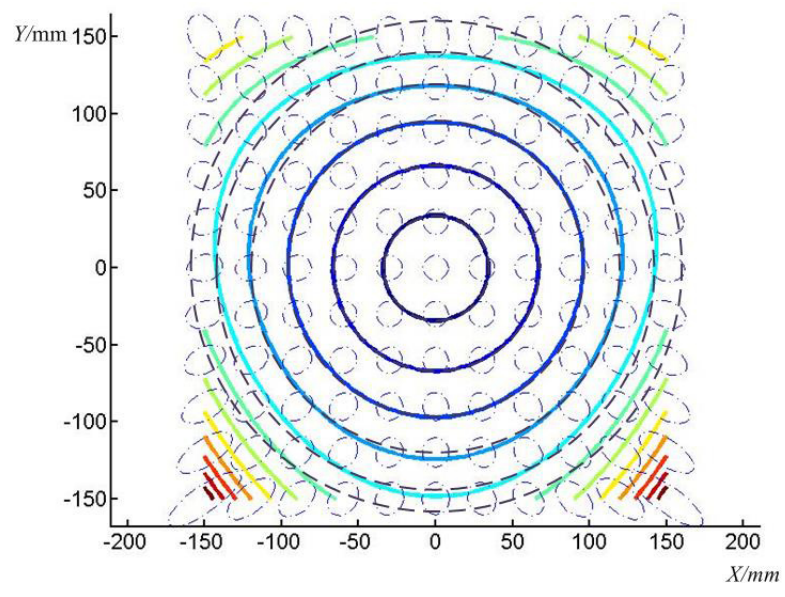

Figure 12. Stiffness contours of the platform in case 1.

Given initial $\theta_{1}$ and $\theta_{2}$ are 75 and $90^{\circ}$, the stiffness contours of the platform within the area of 150 by 150 are plotted, as shown in Fig. 12. The generalized force ellipsoids are used to evaluate the isotropy of the above stiffness matrix. The region where the contours are more intensive has higher stiffness and vice versa. If the shape of the contour approximates a circle, the stiffness of the platform in this circle can be treated as isotropy. In Fig. 12, the contours in the region of 100 by 100 are nearly circular. The generalized force ellipsoids in this region are also rounder than those outside. It appears that the stiffness here is isotropic. With the increasing area, the shapes of the contours and the generalized force ellipsoids are more different from a circle. It illustrates that the feature of stiffness becomes more anisotropic. For further discussion, we take the platform a certain distances away from the center point along $\pm 45, \pm 90, \pm 135,0$ and $180^{\circ}$ axes. Then we release it and let it vibrate. The responses of the displacements of the platform are plotted in Fig. 13. The responses of the displacements from left to right are under the conditions of the given distances of 50,100 and $141 \mathrm{~mm}$. The red dots represent the release position. As the distance 

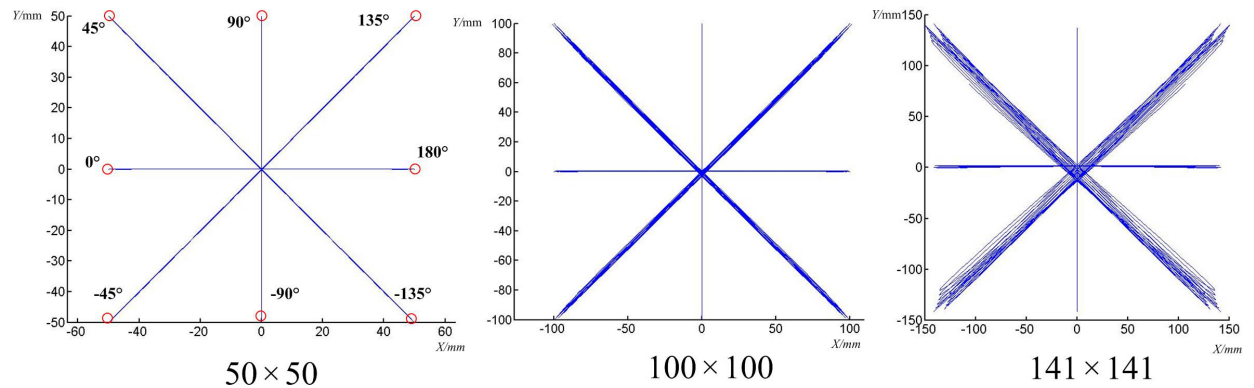

Figure 13. Responses of the displacements of the platform in case 1.

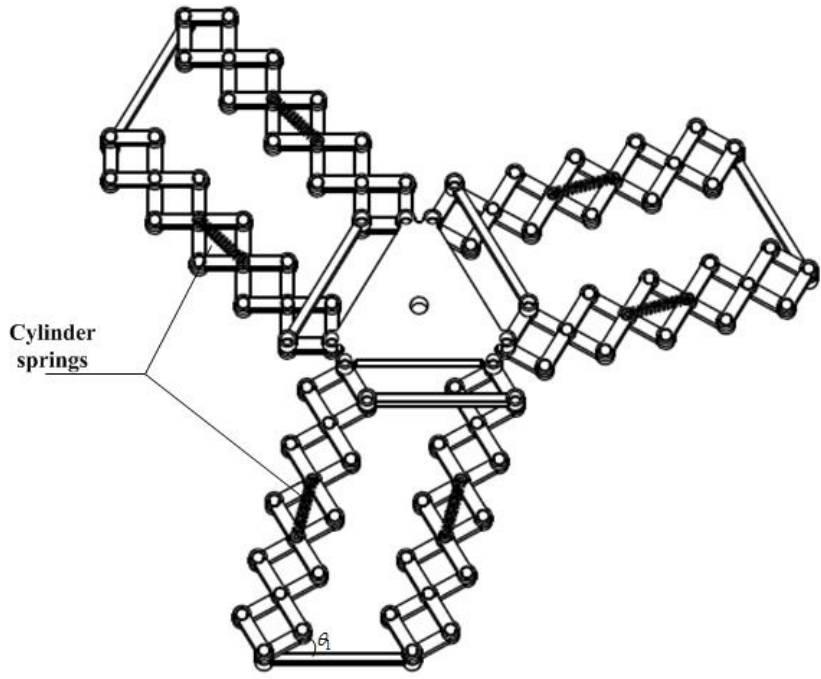

Figure 14. Cylinder springs connecting the vertical joints.

increases, the response of the displacement appears whirling, which means the stiffness is becoming anisotropic. It agrees with the result of Fig. 12.

\subsection{Case 2: cylinder springs connecting the vertical joints}

As shown in Fig. 14, the cylinder springs connect the vertical joints in SLE-Pa legs. Given that the stiffness coefficient of each spring is $k_{\mathrm{v}}$, the free length of the spring is $h_{0}$; the total elastic energy of the mechanism is

$V=\sum_{i=1}^{n} \frac{1}{2} k_{\mathrm{v}}\left({ }^{i} h_{1}-h_{0}\right)^{2}=\sum_{i=1}^{n} \frac{1}{2} k_{\mathrm{v}}\left(\frac{{ }^{i} H_{1}}{s}-h_{0}\right)^{2}$.

Differentiating Eq. (36) to the displacements $p_{x}$ and $p_{y}$, the corresponding generalized force $Q_{x}$ and $Q_{y}$ of the platform can be obtained:

$F_{x}=\sum_{i=1}^{n} k_{\mathrm{v}}\left(\frac{H_{1}}{s}-h_{0}\right) \frac{\cos ^{i} \theta_{1}}{s}$

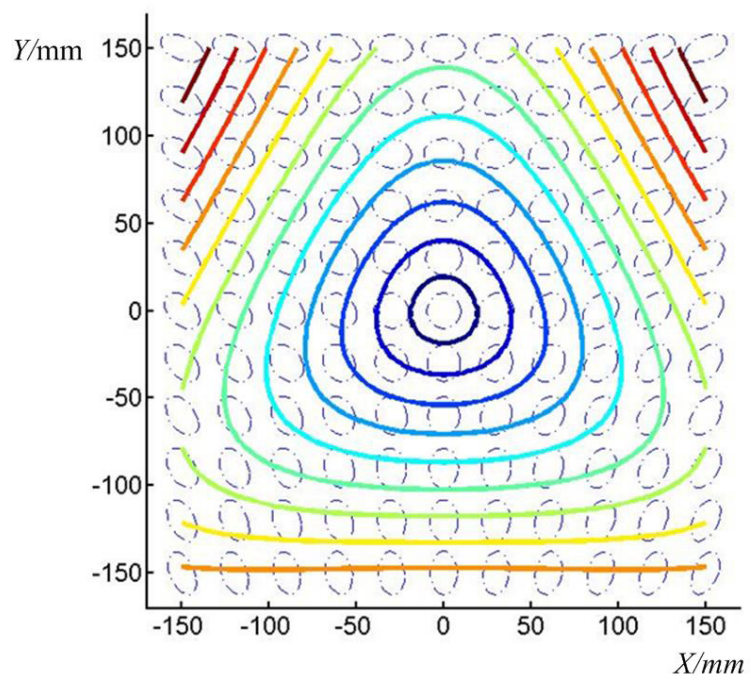

Figure 15. Stiffness contours of the platform in case 2.

$F_{y}=\sum_{i=1}^{n} k_{\mathrm{v}}\left(\frac{{ }^{i} H_{1}}{s}-h_{0}\right) \frac{\sin ^{i} \theta_{1}}{s}$.

The stiffness matrix of the platform can be derived as

$\mathbf{K}_{\mathrm{v}}=\frac{k_{\mathrm{v}}}{s^{2}}\left[\begin{array}{cc}\sum_{i=1}^{n} \cos ^{2^{i}} \theta_{1} & \sum_{i=1}^{n} \cos ^{i} \theta_{1} \sin ^{i} \theta_{1} \\ \sum_{i=1}^{n} \cos ^{i} \theta_{1} \sin ^{i} \theta_{1} & \sum_{i=1}^{n} \sin ^{2^{i}} \theta_{1}\end{array}\right]$,

given that initial $\theta_{1}$ and $\theta_{2}$ are 75 and $90^{\circ}$. In the area of 150 by 150 , the stiffness contours and the generalized force ellipsoids are plotted, as shown in Fig. 15. It may be seen that the shapes of the contours and the generalized force ellipsoids appear very different from circles. Thus, the stiffness of the platform is anisotropic under this circumstance. To test this point further, the platform is taken $141 \mathrm{~mm}$ from the center point and released. The response of free vibration is plotted in Fig. 16. The whirling trajectory proves that the above analysis is correct. 


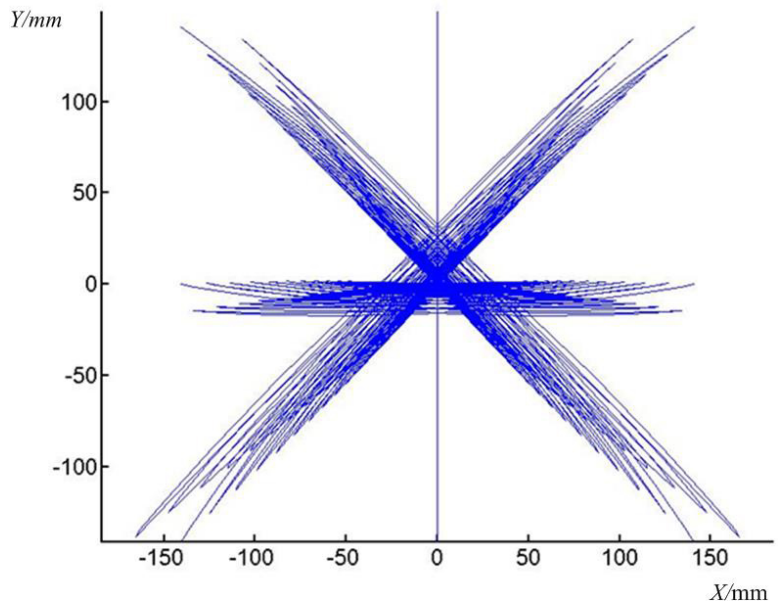

Figure 16. Responses of the displacements of the platform in case 2.

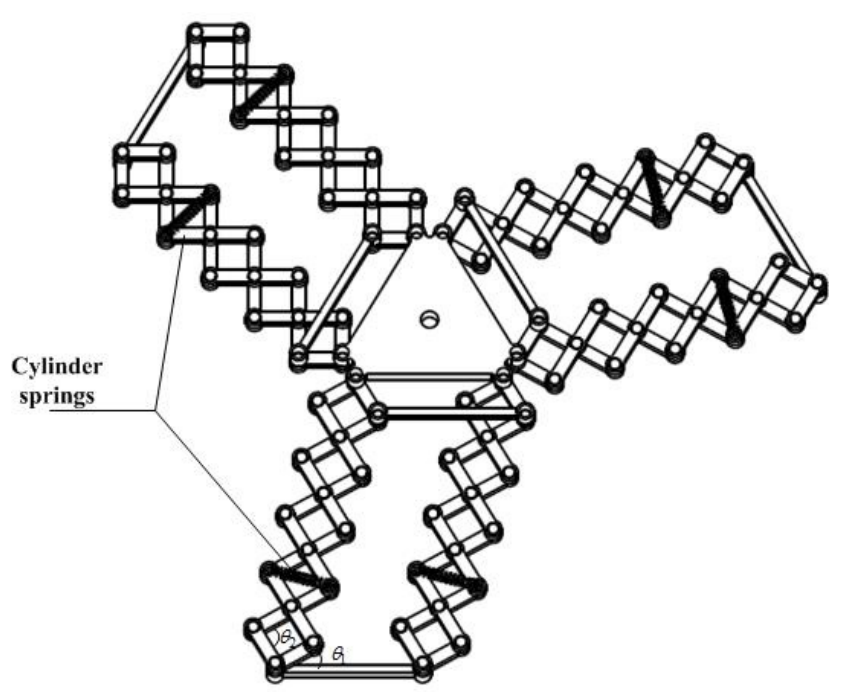

Figure 17. Cylinder springs connecting the horizontal joints.

\subsection{Case 3: cylinder springs connecting the horizontal joints}

As shown in Fig. 17, the corresponding generalized force $Q_{x}$ and $Q_{y}$ of the platform can be obtained by the use of the energy method.

$$
\begin{aligned}
& F_{x}=-\sum_{i=1}^{n} k_{\mathrm{h}} l \sin \frac{{ }^{i} \theta_{2}}{2}\left(2 l \cos \frac{{ }^{i} \theta_{2}}{2}-D_{0}\right) \frac{\partial^{i} \theta_{2}}{\partial p_{x}} \\
& F_{y}=-\sum_{i=1}^{n} k_{\mathrm{h}} l \sin \frac{{ }^{i} \theta_{2}}{2}\left(2 l \cos \frac{{ }^{i} \theta_{2}}{2}-D_{0}\right) \frac{\partial^{i} \theta_{2}}{\partial p_{y}}
\end{aligned}
$$

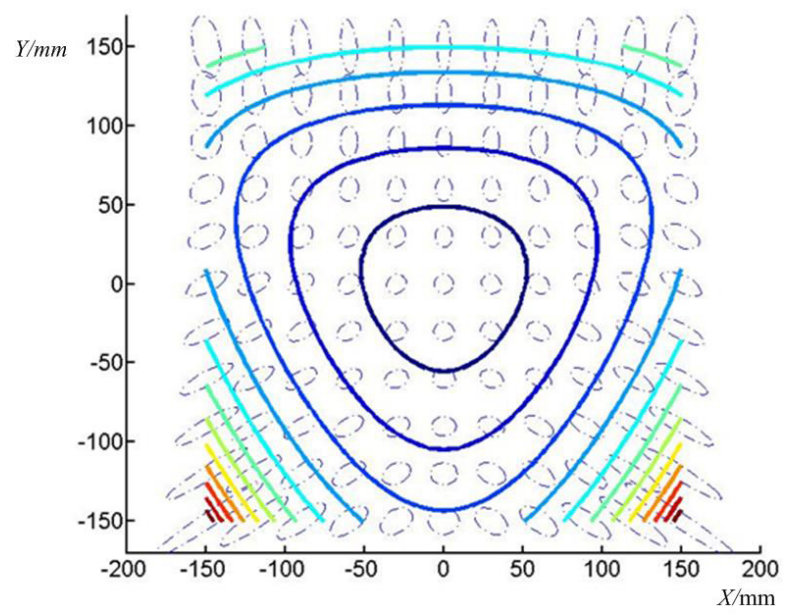

Figure 18. Stiffness contours of the platform in case 3.

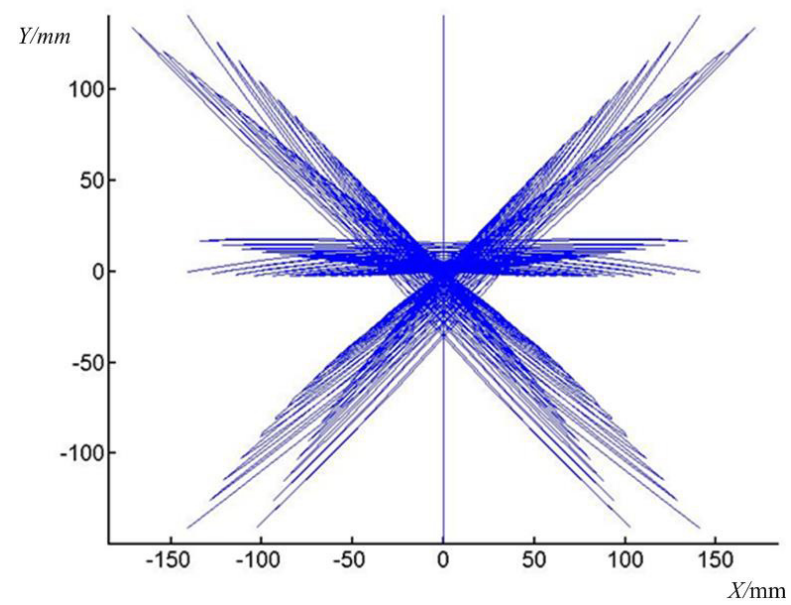

Figure 19. Responses of the displacements of the platform in case 3 .

where $k_{\mathrm{h}}$ is the stiffness coefficient of each spring. The stiffness matrix of the platform can be derived as

$$
\mathbf{K}_{\mathrm{h}}=-\frac{k_{\mathrm{h}}}{s^{2} l}\left[\begin{array}{c}
\sum_{i=1}^{n}\left(l \cos ^{i} \theta_{2}-\frac{1}{2} D_{0} \cos \frac{{ }^{i} \theta_{2}}{2}\right) \frac{\cos _{2}^{i} \theta_{1}}{\cos ^{2} \frac{\theta_{2}}{2}} \\
\sum_{i=1}^{n}\left(l \cos ^{i} \theta_{2}-\frac{1}{2} D_{0} \cos \frac{{ }^{i} \theta_{2}}{2}\right) \frac{\cos ^{i} \theta_{1} \sin ^{i} \theta_{1}}{\cos ^{2} \frac{\theta_{2}}{2}} \\
\sum_{i=1}^{n}\left(l \cos ^{i} \theta_{2}-\frac{1}{2} D_{0} \cos \frac{{ }^{i} \theta_{2}}{2}\right) \frac{\cos ^{i} \theta_{1} \sin ^{i} \theta_{1}}{\cos ^{2} \frac{\theta_{2}}{2}} \\
\sum_{i=1}^{n}\left(l \cos ^{i} \theta_{2}-\frac{1}{2} D_{0} \cos \frac{{ }^{i} \theta_{2}}{2}\right) \frac{\sin ^{2}{ }^{2} \theta_{1}}{\cos ^{2} \frac{i_{2}}{2}}
\end{array}\right]
$$

The stiffness contours and the generalized force ellipsoids are drawn in Fig. 18. The feature of the stiffness is anisotropic. The response of free vibration in Fig. 19 also proves this result. 


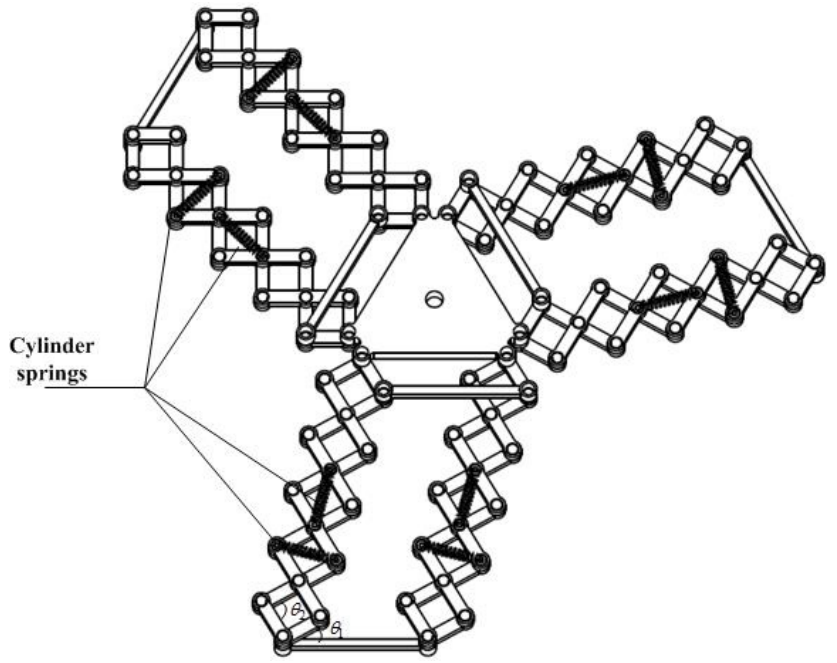

Figure 20. Cylinder springs connecting vertical joints and horizontal joints.

\subsection{Case 4: cylinder springs connecting vertical joints and horizontal joints}

As shown in Fig. 20, the cylinder springs connect the vertical and horizontal joints in SLE-Pa legs. The stiffness coefficient of each spring is entirely identical, i.e., $k_{\mathrm{v}}=k_{\mathrm{h}}$. The stiffness of the platform can be regarded as the sum of Eqs. (38) and (40). The contours and the generalized force ellipsoids can be drawn, and the response of free vibration is displayed in Figs. 21 and 22. It is found that the stiffness of the platform appears to be isotropic in this compound connection. By the comparison of Figs. 15 and 18, the reason is found that the convexities of the contours in these two figures are the opposite of one another. This means that the recovery forces of the platform in these two cases may be complementary, which results in the stiffness being isotropic. The responses of the free vibration also illustrate this point. Through this case, it may be concluded that the isotropic stiffness can be synthesized by different anisotropic stiffness cases according to the feature of the stiffness contours.

\section{Approximation of stiffness coefficient}

Since the SLE-Pa legs are distributed around the center of the mechanism, ${ }^{1} \theta_{1},{ }^{2} \theta_{1}, \ldots,{ }^{n} \theta_{1}$ form an arithmetic progression if the displacement of the platform is small.

${ }^{i} \theta_{1}={ }^{1} \theta_{1}+\frac{2 \pi}{n}(i-1) \quad i=1,2, \ldots, n$

${ }^{i} \theta_{2}$ in each leg seems to be equal.

${ }^{1} \theta_{2}={ }^{2} \theta_{2}=\ldots={ }^{n} \theta_{2}$

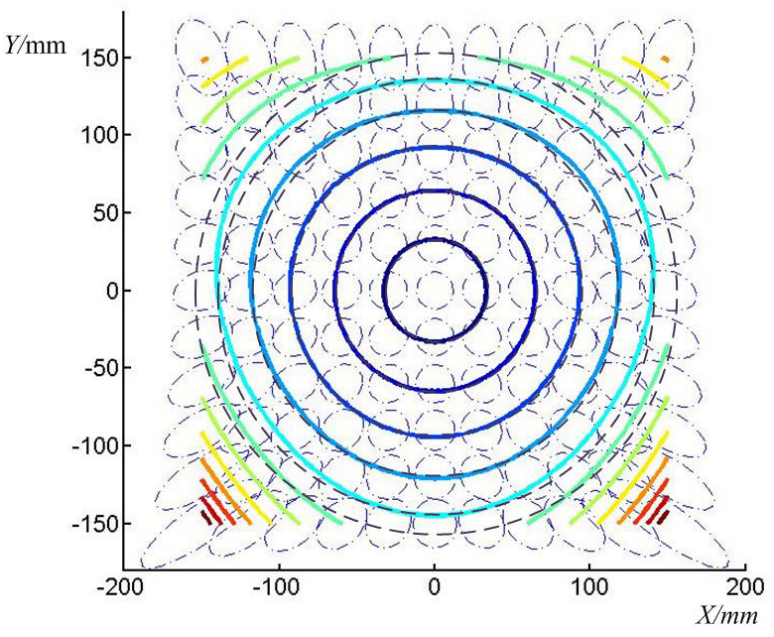

Figure 21. Stiffness contours of the platform in case 4.

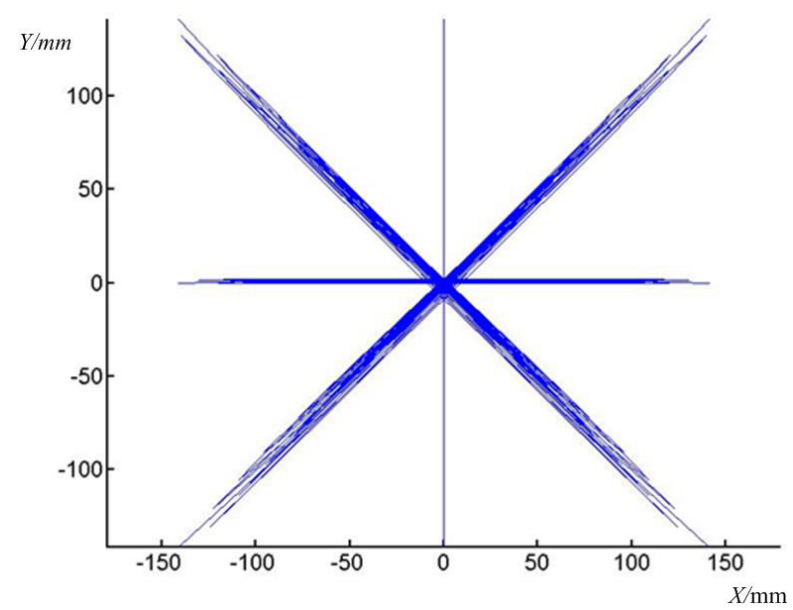

Figure 22. Responses of the displacements of the platform in case 4.

With the following condition

$$
\begin{aligned}
& \sum_{i=1}^{n} \cos ^{2} \theta_{i}=\frac{1}{2} n \quad \sum_{i=1}^{n} \sin ^{2} \theta_{i}=\frac{1}{2} n \\
& \sum_{i=1}^{n} \sin \theta_{i} \cos \theta_{i}=0,
\end{aligned}
$$

the approximation of the stiffness coefficient for case 1 in Sect. 4 can be deduced as

$$
\mathbf{K}_{\theta}=\frac{k_{\theta}}{(s l)^{2}}\left[\begin{array}{cc}
\sum_{i=1}^{n} \frac{\cos ^{2}{ }^{i} \theta_{1}}{\cos ^{2} \frac{\theta_{2}}{2}} & \sum_{i=1}^{n} \frac{\cos ^{i} \theta_{1} \sin ^{i} \theta_{1}}{\cos ^{2} \frac{\theta_{2}}{2}} \\
\sum_{i=1}^{n} \frac{\cos ^{i} \theta_{1} \sin ^{i} \theta_{1}}{\cos ^{2} \frac{\theta_{2}}{2}} & \sum_{i=1}^{n} \frac{\sin ^{2} \theta_{1}}{\cos ^{2} \frac{\theta_{2}}{2}}
\end{array}\right]
$$




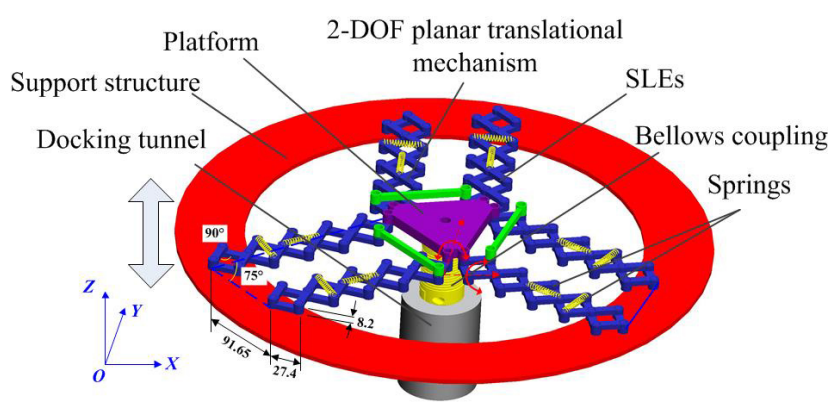

Figure 23. Passive docking device.

$$
=\frac{k_{\theta}}{(s l)^{2} \cos ^{2} \frac{0}{2} \theta_{2}}\left[\begin{array}{cc}
\frac{1}{2} n & 0 \\
0 & \frac{1}{2} n
\end{array}\right] .
$$

In the same way, the approximation of the stiffness coefficient for cases 2 and 3 is

$$
\begin{aligned}
& \mathbf{K}_{\mathrm{v}}=\frac{k_{\mathrm{v}}}{s^{2}}\left[\begin{array}{cc}
\sum_{i=1}^{n} \cos ^{2^{i}} \theta_{1} & \sum_{i=1}^{n} \cos ^{i} \theta_{1} \sin ^{i} \theta_{1} \\
\sum_{i=1}^{n} \cos ^{i} \theta_{1} \sin ^{i} \theta_{1} & \sum_{i=1}^{n} \sin ^{2^{i}} \theta_{1}
\end{array}\right] \\
& =\frac{k_{\mathrm{v}}}{s^{2}}\left[\begin{array}{cc}
\frac{1}{2} n & 0 \\
0 & \frac{1}{2} n
\end{array}\right] \text {, } \\
& \mathbf{K}_{\mathrm{h}}=-\frac{k_{\mathrm{h}}}{s^{2} l} \frac{\left(l \cos ^{0} \theta_{2}-\frac{1}{2} D_{0} \cos \frac{{ }^{0} \theta_{2}}{2}\right)}{\cos ^{2} \frac{\theta_{2}}{2}}\left[\begin{array}{cc}
\frac{1}{2} n & 0 \\
0 & \frac{1}{2} n
\end{array}\right] \\
& =\frac{k_{\mathrm{h}}}{s^{2}} \tan ^{2} \frac{0 \theta_{2}}{2}\left[\begin{array}{cc}
\frac{1}{2} n & 0 \\
0 & \frac{1}{2} n
\end{array}\right] \text {. }
\end{aligned}
$$

The approximation of the stiffness coefficient can be used for a preliminary estimate of some parameters, i.e., recovery force, vibration frequency and others, before designing the mechanism. It is found that the approximation of the stiffness coefficient in cases 1 and 3 is related to ${ }^{0} \theta_{2}$. It means that the stiffness of the platform can be adjusted by changing ${ }^{0} \theta_{2}$. If $k_{\mathrm{v}}=k_{\mathrm{h}}$ and ${ }^{0} \theta_{2}=90^{\circ}$, the stiffness in case 2 and 3 is equal.

\section{Design of passive docking device}

These novel 2-DOF planar translational mechanisms assembled by SLE-Pa legs are applied to the design of a passive docking device, which can be used for joining pipes in a water sample collection system. As shown in Fig. 23, the passive docking device comprises support structure, 2DOF planar translational mechanism, bellows coupling and

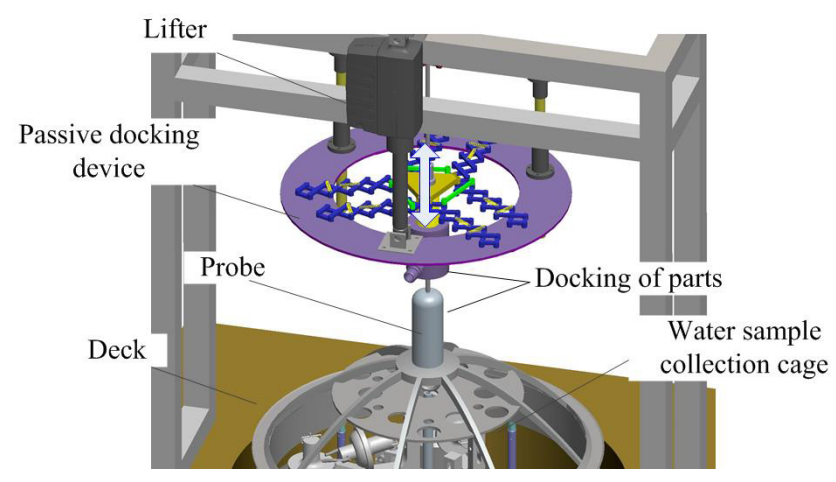

Figure 24. Docking diagram.

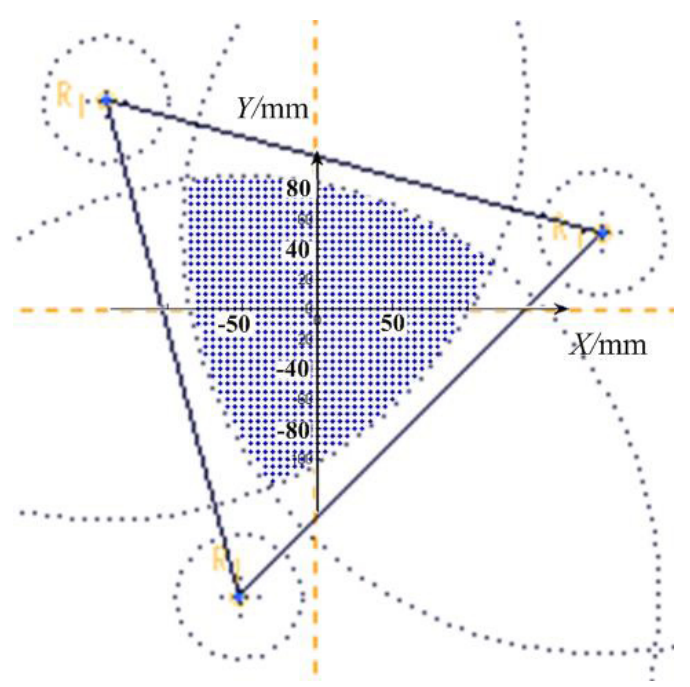

Figure 25. Work space of 2-DOF planar translational mechanism.

a docking tunnel. The bellows coupling provides $x$ - and $y$ axes rotational DOFs. The 2-DOF planar translational mechanism provides $x$ - and $y$-axes translational DOFs. The support structure is taken by a lifter, which moves along the $z$ axis. Thus, the docking tunnel has a total of 5 DOFs, which has the ability to adapt to the misalignments in the docking procedure. In addition, for the purposes of a passive docking device capable of an isotropic recovery force, the cylinder springs are installed to connect vertical joints and horizontal joints. The stiffness coefficient of each cylinder spring is $0.3 \mathrm{~N} \mathrm{~mm}^{-1}$. The total mass of the platform, bellows coupling and docking tunnel is $1.247 \mathrm{~kg}$.

As shown in Fig. 24, the water sample collection cage is fixed to the deck by the holding mechanism when it is pulled up from the sea. There is a docking probe at the top of the water sample collection cage. When the cage is put in place, the lifter takes the passive docking device and allows it to descend to approach the probe. In the docking procedure, the probe slowly slides into the tunnel. The 2-DOF planar translational mechanism and bellows coupling enable the tunnel to possess 4 DOFs in order to compensate for the misalign- 


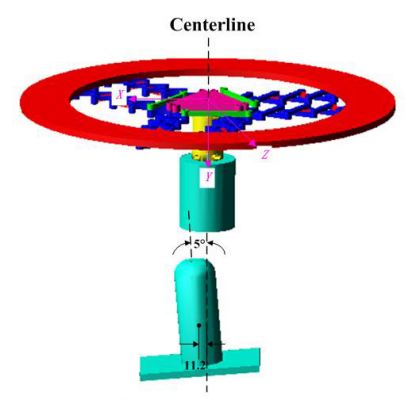

(a) Approaching

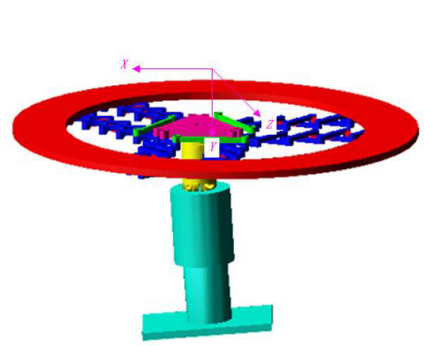

(c) Docked

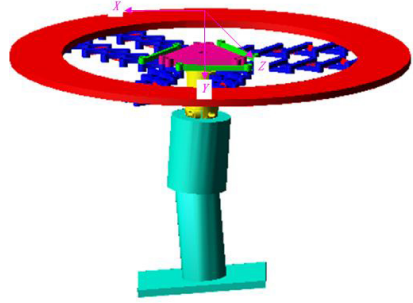

(b) Accessing

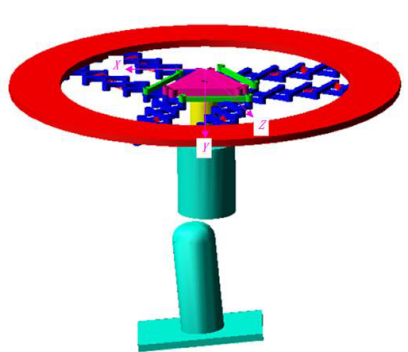

(d) Separating

Figure 26. Docking procedure.

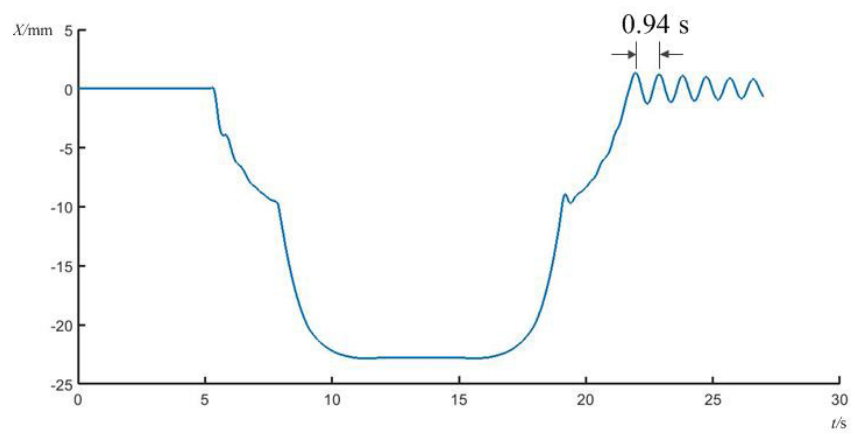

Figure 27. $x$-axis displacement of the platform.

ments between the tunnel and probe. When the probe has entirely entered the tunnel, the sample water in the collection cage is drawn out. After this operation, the lifter takes the passive docking device and moves it up to the original place.

In the 2-DOF planar translational mechanism, the length of half an SLE is $l=27.4 \mathrm{~mm}$, and the width is $w=8.2 \mathrm{~mm}$. The number of SLEs in a single SLE limb is $s=5$. The initial angles $\theta_{1}$ and $\theta_{2}$ are 75 and $90^{\circ}$, respectively. The minimum length of an SLE-Pa leg is $H_{\mathrm{lb}} \approx s \cdot w=41 \mathrm{~mm}$. The maximum length of an SLE-Pa leg is $H_{\mathrm{ub}} \approx 2 \cdot s \cdot l=274 \mathrm{~mm}$. The ratio of $H_{\mathrm{ub}}$ to $H_{\mathrm{lb}}$ is 6.68 , which is larger than $\sqrt{3}$. According to Sect. 3, the shape of the work space belongs to type I(a), as shown in Fig. 25.

The docking procedure is simulated by Adams, as shown in Fig. 26. Assume that the center of the probe has an offset distance $11.2 \mathrm{~mm}$ from the centerline and a slip angle $5^{\circ}$ off the horizon. At the beginning, the passive docking device

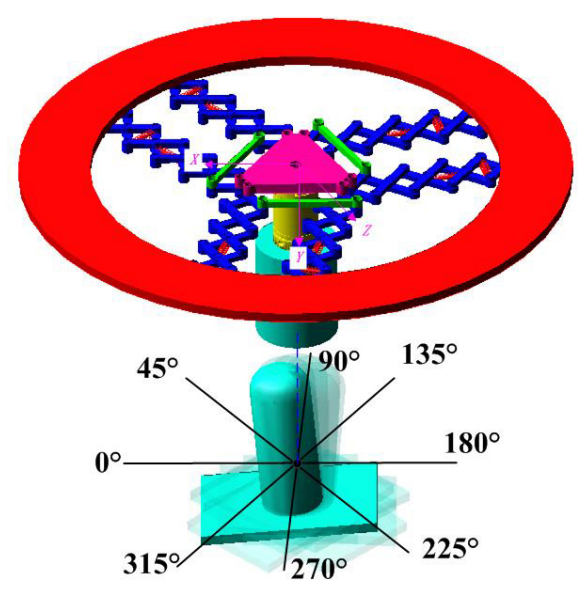

Figure 28. Eight rotation angles of the probe.

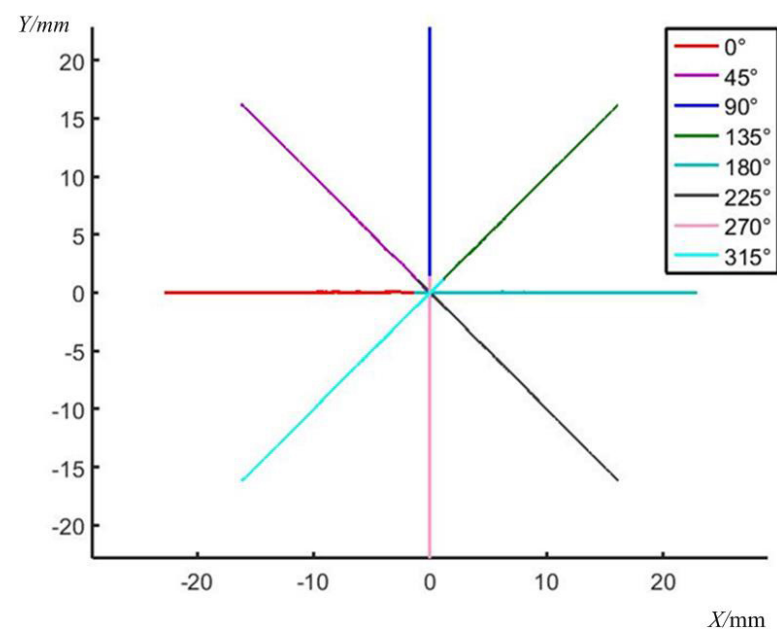

Figure 29. Eight displacement trajectories of the platform.

descends at a velocity of $10 \mathrm{~mm} \mathrm{~s}^{-1}$ to dock with the probe. When complete, the docking device stops for $3 \mathrm{~s}$. Then, it ascends at a velocity of $10 \mathrm{~mm} \mathrm{~s}^{-1}$ until it reaches the original position. The simulation proved that the 2-DOF translational mechanism is able to produce a smooth motion in $x-y$ plane.

The $x$-axis displacement of the platform of the 2-DOF translational mechanism is measured in the simulation, as shown in Fig. 27. It may be seen that the platform generates vibration after the docking tunnel and the probe have been separated. This is because of the cylinder springs in the mechanism, which result in the recovery force. The period of vibration is roughly measured as $0.94 \mathrm{~s}$ in Fig. 27.

We can also calculate the period of vibration by the approximation of the stiffness coefficient derived in Sect. 5. By the utilization of Eqs. (45) and (46) and the consideration of two vertical and horizontal springs in each leg, the approximation of the stiffness coefficient can be obtained.

$k_{\mathrm{E}}=2 \times\left(\frac{n}{2} \frac{k_{\mathrm{v}}}{s^{2}}+\frac{n}{2} \frac{k_{\mathrm{h}}}{s^{2}}\right)=0.072 \mathrm{Nmm}^{-1}$. 


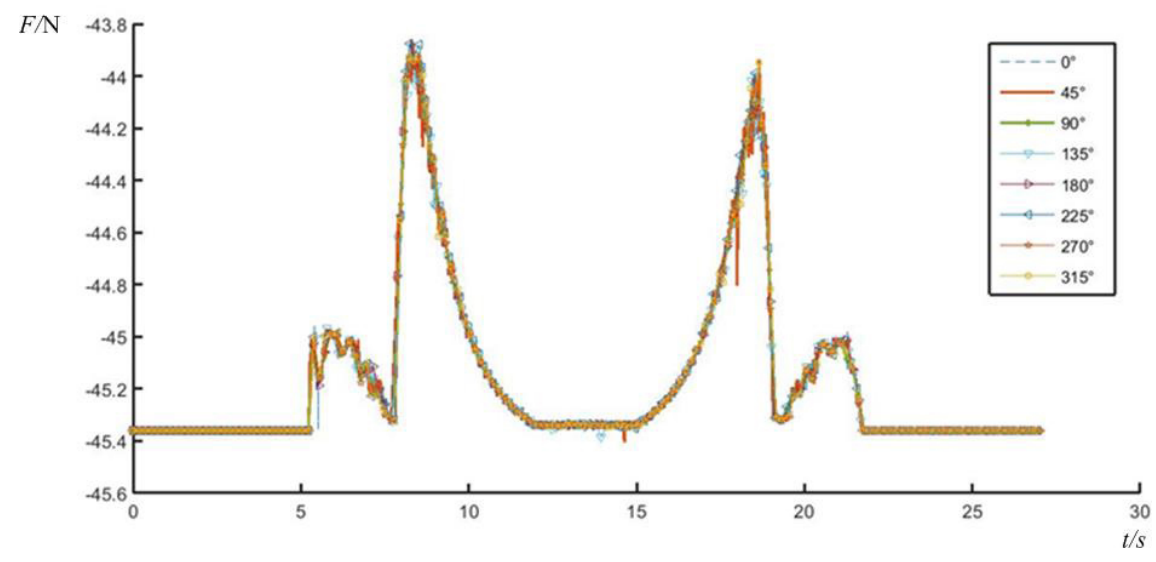

Figure 30. Actuator forces in eight cases.
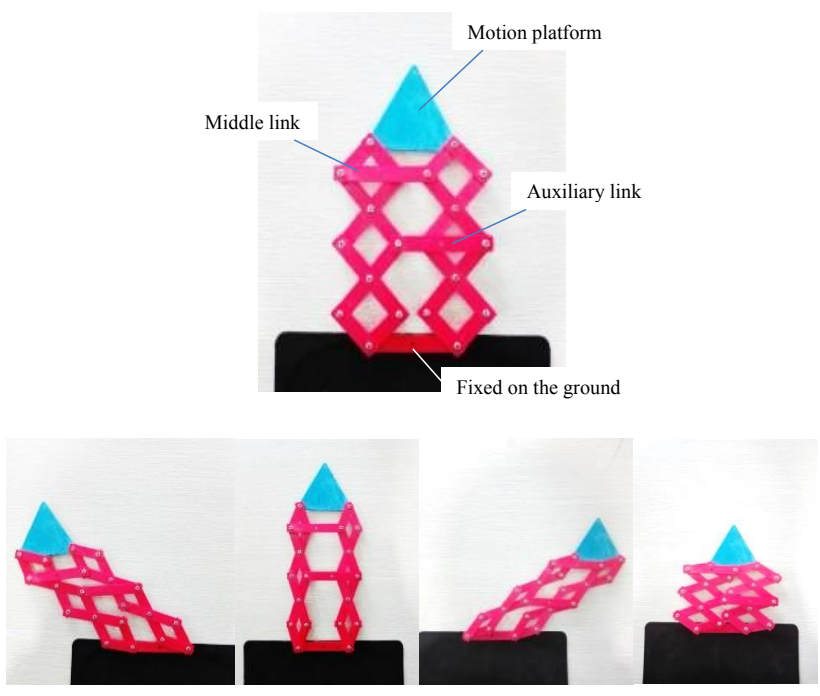

Figure 31. Prototype of one SLE-Pa leg.

The period of vibration can be then calculated as

$T=\frac{2 \pi}{\omega}=2 \pi \sqrt{\frac{m}{k_{\mathrm{E}}}}=0.827 \mathrm{~s}$.

This result is very close to the one of the simulation. It proves the correction of the above analysis. To illustrate the isotropy of the recovery force, the probe is rotated around the centerline to change its position and posture. The rotation angles form a tolerance of $45^{\circ}$ arithmetic progression from 0 to $315^{\circ}$, as shown in Fig. 28. The docking simulation is conducted for each rotation angle, i.e., in total eight cases that need to be solved.

The eight displacement trajectories of the platform in the $x-z$ plane are calculated and plotted in Fig. 29. It shows that all the trajectories are straight lines and there is no whirling. The actuator forces in eight cases which drive the passive docking device up and down are also measured and plotted, as shown in Fig. 30. The eight force curves are almost the same although the positions and postures of the probes are different. Through Figs. 29 and 30, it can be concluded that the stiffness of the passive docking device in the docking procedure is isotropic. The prototype of one SLE-Pa leg is also manufactured, as shown in Fig. 31. The auxiliary link in the prototype provides overconstraint which is to enhance the parallel constraint for the two SLE limbs.

\section{Conclusion}

A novel 2-DOF planar translational mechanism comprising SLE-Pa legs is proposed in this paper. The SLE-Pa leg consists of two identical SLE limbs. The two corresponding nodes in the two SLE limbs are hinged by one middle link. According to three different hinged positions of the middle link, the mobilities and kinematics are discussed by screw theory. The result shows that if the middle link connects two corresponding corner joints of the SLE limbs, these two SLE limbs are always parallel and the SLE-Pa leg has 2 pure translational degrees of freedom. Based on that, a novel 2-DOF translational mechanism is invented. The kinematics equations and the work spaces of the platform are given. For the purpose of this mechanism capable of recovery force, the elastic elements are installed for this mechanism. Through the analysis of the stiffness contours and the generalized force ellipsoids, the isotropy of the stiffness of the platform has been identified. By applying the above results, the passive docking device with the isotropic recovery force is invented. The docking procedure is simulated by Adams, which proves the feasibility of the 2-DOF translational mechanism and the isotropy of the device. Moreover, the prototype of one SLE-Pa leg is manufactured and presented.

Data availability. The videos of the simulation are provided in the Supplement. 


\section{The Supplement related to this article is available online at https://doi.org/10.5194/ms-8-179-2017-supplement.}

Competing interests. The authors declare that they have no conflict of interest.

Acknowledgements. This project was supported by the National Natural Science Foundation of China (nos. 51675318, 51575329, 61525305).

Edited by: Chin-Hsing Kuo

Reviewed by: two anonymous referees

\section{References}

Bai, G., Liao, Q., Li, D., and Wei, S.: Synthesis of scaling mechanisms for geometric figures with angulated-straight elements, Proc IMechE Part C: J Mechanical Engineering Science, 227, 2795-2809, 2013.

Dai, J. S. and Rees, J. J.: Mobility in metamorphic mechanisms of foldable/erectable kinds, ASME Transaction Journal of Mechanical Design, 121, 375-382, 1999.

Ding, X. L., Yang, Y., and Dai, J. S.: Topology and Kinematic Analysis of Color-Changing Ball, Mech. Mach. Theory, 46, 67-81, 2011.

Dong, J., Yuan, C., Stori, J. A., and Ferreira, P. M.: Development of a High-speed 3-axis Machine Tool using a Novel Parallelkinematics X-Y Table, International Journal of Machine Tools and Manufacture, 44, 1355-1371, 2004.

Escrig, F. and Valcarcel, J. P.: Geometry of expandable space structures, International Journal of Space Structures, 8, 71-84, 1993.

Escrig, F., Valcarcel, J. P., and Sanchez, J.: Deployable cover on a swimming pool in Seville, Bulletin of the International Association for Shell and Spatial Structures, 37, 39-70, 1996.

Huang, T., Li, Z., Li, M., Chetwynd, D. G., and Gosselin, C. M.: Conceptual design and dimensional synthesis of a novel 2DOF translational parallel robot for pick-and-place operations, J. Mech. Design, 126, 449-455, 2004.

Huang, T., Liu, S., Mei, J., and Chetwynd, D. G.: Optimal design of a 2-DOF pick-and-place parallel robot using dynamic performance indices and angular constraints, Mech. Mach. Theory, 70, 246-253, 2013.

Kaveh, A. and Davaran, A.: Analysis of pantograph foldable structure, Comput. Struct., 59, 131-140, 1996.
Kaveh, A., Jafarvand, A., and Barkhordari, M. A.: Optimal design of pantograph foldable structures, International Journal of Space Structures, 14, 295-302, 1999.

Kim, H. S.: Development of Two Types of Novel Planar Translational Parallel Manipulators by using Parallelogram Mechanism, Journal of the Korean Society of Precision Engineering, 24, 50 57, 2007.

Langbecker, T.: Kinematic Analysis of Deployable Scissor Structures, International Journal of Space Structures, 14, 1-15, 1999.

Liu, X. J. and Wang, J.: Some New Parallel Mechanisms Containing the Planar Four-Bar Parallelogram, Int. J. Robot. Res., 22, 717732, 2003.

Liu, X. J., Wang, Q. M., and Wang, J.: Kinematics, Dynamics and Dimensional Synthesis of a Novel 2-DOF Translational Manipulator, J. Intell. Robot. Syst., 41, 205-224, 2004.

Liu, X. J., Wang, J., and Pritschow, G.: On the Optimal Kinematic Design of the PRRRP 2-DOF Parallel Mechanism, Mech. Mach. Theory, 41, 1111-1130, 2006.

Lu, S., Zlatanov, D., and Ding, X.: Approximation of Cylindrical Surfaces with Deployable Bennett Networks, Journal of Mechanisms and Robotics, 9, 021001, https://doi.org/10.1115/1.4035801, 2017.

Pham, V. N. and Kim, H. S.: Dynamics Analysis of a 2-DOF Planar Translational Parallel Manipulator, Journal of the Korean Society of Manufacturing Technology Engineers, 22, 185-191, 2013.

Rosenfeld, Y. and Logcher, R. D.: New concepts for deployablecollapsable structures, International Journal of Space Structures, 3, 20-32, 1988.

Wei, G., Ding, X., and Dai, J S.: Mobility and geometric analysis of the Hoberman switch-pitch ball and its variant, Journal of Mechanisms and Robotics, 2, 191-220, 2010.

Wu, J., Wang, J., Li, T., and Wang, L.: Analysis and application of a 2-DOF planar parallel mechanism, J. Mech. Design, 129, 434 437, 2007.

You, Z. and Pellegrino, S.: Foldable Bar Structures, Int. J. Solids Struct., 34, 1825-1847, 1997.

Zhao, J. S., Wang, J. Y., Chu, F., Feng, Z. J., and Dai, J. S.: Structure synthesis and statics analysis of a foldable stair, Mech. Mach. Theory, 46, 998-1015, 2011. 